\title{
The general inefficiency of batch training for gradient descent learning
}

\author{
D. Randall Wilson ${ }^{\mathrm{a}, *}$, Tony R. Martinez ${ }^{\mathrm{b}, 1}$ \\ ${ }^{a}$ Fonix Corporation, 180 West Election Road Suite 200, Draper, UT, USA \\ ${ }^{\mathrm{b}}$ Computer Science Department, 3361 TMCB, Brigham Young University, Provo, UT 84602, USA
}

Received 10 July 2001; revised 8 April 2003; accepted 8 April 2003

\begin{abstract}
Gradient descent training of neural networks can be done in either a batch or on-line manner. A widely held myth in the neural network community is that batch training is as fast or faster and/or more 'correct' than on-line training because it supposedly uses a better approximation of the true gradient for its weight updates. This paper explains why batch training is almost always slower than on-line training — often orders of magnitude slower-especially on large training sets. The main reason is due to the ability of on-line training to follow curves in the error surface throughout each epoch, which allows it to safely use a larger learning rate and thus converge with less iterations through the training data. Empirical results on a large (20,000-instance) speech recognition task and on 26 other learning tasks demonstrate that convergence can be reached significantly faster using on-line training than batch training, with no apparent difference in accuracy.
\end{abstract}

(C) 2003 Elsevier Ltd. All rights reserved.

Keywords: Batch training; On-line training; Gradient descent; Backpropagation; Learning rate; Optimization; Stochastic approximation; Generalization

\section{Introduction}

Neural networks are often trained using algorithms that approximate gradient descent. Gradient descent learning (also called steepest descent) can be done using either a batch method or an on-line method. In batch training, weight changes are accumulated over an entire presentation of the training data (an epoch) before being applied, while on-line training updates weights after the presentation of each training example (instance). Another alternative is sometimes called mini-batch (Sarle, 2002), in which weight changes are accumulated over some number $u$ of instances before actually updating the weights. Using an update frequency (or batch size) of $u=1$ results in on-line training, while $u=N$ results in batch training, where $N$ is the number of instances in the training set. In each case a learning rate, $r$, is used to adjust the size of weight changes.

Most neural network researchers would agree that the question of whether batch or on-line training is faster and/or

\footnotetext{
* Corresponding author. Tel.: + 1-801-446-1697.

E-mail address: randy@axon.cs.byu.edu (D.R. Wilson).

1 Tel.: +1-801-422-6464; fax: + 1-801-422-7775.
}

'better' has been settled. However, it is interesting to note that people have unknowingly 'settled' upon two opposing views. Some believe that since batch uses the 'true' gradient direction for its updates, it is more 'correct,' and some researchers claim or imply that batch training is faster. Others note that in practice on-line training appears to train more quickly and produce better results than batch training.

This paper explains why on-line training can be expected to be at least as accurate as well as faster than batch training, and demonstrates empirically that on-line training is often orders of magnitude faster than batch training, especially on large training sets.

To briefly overview the main points of this paper, batch training is able to calculate the direction of the true gradient, but it does not know how far it can safely go in that direction before the gradient changes direction (or begins to go back 'uphill'). The true gradient can curve considerably on its way to a minimum, but batch training can only take one step for each epoch, and each step is in a straight line.

As the size of the training set grows, the accumulated weight changes for batch training become large. This leads batch training to use unreasonably large steps, which in turn leads to unstable learning and to the overshooting of curves 
and local minima in the error landscape. Therefore, a reduction in the size of the learning rate (or, equivalently, using the average weight change instead of the sum) is required for batch training to be stable as the size of the training set grows. However, this means that the larger the training set is, the more computation is required for a weight change of the same magnitude.

On the other hand, on-line training uses the 'local' gradient of each training instance to determine what direction to go. These local gradients can be noisy and contradict each other, but will on average move in the direction of the true gradient. Since on-line training updates weights after each instance, it is able to follow curves in the gradient during the course of an epoch. It can also handle any size of training set without having to reduce the learning rate.

Section 2 surveys the neural network literature to examine what is currently being taught about on-line and batch training. Some of the references make false claims or use misleading examples, while others appear entirely accurate. None of the literature surveyed, however, mentions the ability of on-line training to learn faster as a result of being able to follow the gradient more closely during an epoch and thus use a larger learning rate.

Section 3 explains the above arguments in more detail, describes each training method in terms of optimization theory, and gives several intuitive interpretations of batch and on-line training. It discusses how on-line and batch are similar in order to focus in on what is really different between the methods and how this difference gives on-line training such an advantage.

Some readers may find that Section 3 contains a bit of redundancy, in terms of explaining the same thing in several different ways. However, it is the experience of the authors that some neural network researchers have a difficult time overcoming long-held misunderstandings in this area (while others understand quickly and wonder what all the confusion is about). Hopefully by presenting the arguments in several ways there will remain little room for misunderstanding.

Section 4 presents empirical results of training multilayer perceptrons using error backpropagation with a variety of learning rates on 26 classification tasks from the UCI Machine Learning Database Repository (Blake \& Merz, 1998), with both on-line and batch training. It also compares on-line, batch and mini-batch training on a speech recognition task with a large training set of 20,000 instances. The results strongly support the claim that online training is faster and at least as accurate as batch training, especially for large training sets. The speech recognition experiments also show that the larger the batch size is, the more on-line training outperforms batch training.

Section 4 also explains why using clusters of machines training a single neural network in parallel—which requires batch or mini-batch training-is likely to be slower (as well as more expensive) than using on-line training on a single machine to do the training alone.

Section 5 summarizes the conclusions from these results, which basically are that there are strong disadvantages and no apparent real advantages to using batch training, even for parallel training, and that on-line training should therefore be used in practical gradient descent training situations.

\section{Survey of neural network literature}

This section surveys the neural network literature to examine what is currently being taught about on-line and batch training. As evidenced from the literature and from recent discussions with various researchers, many are still of the opinion that batch training is as fast or faster and/or more correct than on-line training. Unfortunately, this belief may lead those who use gradient descent algorithms in practice to waste vast amounts of computational resources training neural networks inefficiently.

Comparisons between batch and on-line training in the literature have often been misleading. They tend to use very small training sets for which the problems with batch training are less apparent. They have also almost always used the same learning rate for batch and on-line training when comparing the two. While that might seem like the right thing to do in order to make a fair comparison, it misses the point that on-line training can safely use a larger learning rate than batch training, and can thus train more quickly. For large training sets, batch training is often completely impractical due to the minuscule learning rate required (either explicitly, or implicitly due to using the average weight update for each epoch, which effectively divides the learning rate by the number of training instances). This can result in taking hundreds or thousands of times as long to converge as on-line training.

\subsection{Implying that batch is better}

Authors often imply that batch training is at least theoretically superior to on-line training because of its use of the true gradient. Principe, Euliano, and Lefebvre (2000, p. 26, 27), for example, say that batch training "will follow the gradient more closely" than on-line training, which they say "will zig-zag around the gradient direction." These statements are reasonably true when the learning rate is small enough to keep the true gradient fairly constant during each epoch. In such cases, on-line training will fluctuate ('zig-zag') around the (fairly straight) gradient during an epoch, but will end up at essentially the same point at the end of an epoch as batch training does. (Actually, batch training fluctuates just as much during an epoch if the accumulated weight changes are observed, but fluctuates around the tangent line.)

When the learning rate is larger, however, the weights for on-line training will fluctuate around the true (current, often 
curving) gradient during an epoch, while batch will move in a straight line (i.e. in the direction of the gradient at the original location) even if the gradient curves. In this case online training will actually follow the gradient more closely, as illustrated and explained further in Section 3.

They also say that "both converge to the same minimum," which again is true for a sufficiently small learning rate but can be untrue for larger learning rates, since each method will experience a different kind of variation from the true gradient, which can lead them down different paths.

Fausett (1994) alludes to mini-batch and batch training and says "this procedure has a smoothing effect on the correction terms. In some cases, this smoothing may increase the chances of convergence to a local minimum." This reference does not mention the need for reducing the learning rate when accumulating changes over multiple instances, however, and the hypothesis of increased chance of convergence for batch or mini-batch is contradicted by the results in Section 4. In fact, in terms of generalization accuracy at the end of each training epoch, our experiments found that on-line training was much more 'smooth' than mini-batch or batch.

\subsection{Claims that batch is as fast or faster}

Several authors agreed with our findings that on-line training learns more quickly than batch (Bengio, 1991, 1996; Bouttou, Fogelman-Soulie, Blanchet, \& Lienard, 1990). However, many others made no mention of on-line training's faster learning, and there was no mention in the surveyed literature of the ability of on-line training to safely use a larger learning rate than batch due to the way it follows the curving gradient throughout each epoch.

The reason for these oversights is likely that comparisons between batch and on-line training in the literature have almost always used the same learning rate for both methods, i.e. one small enough to allow both to converge. While this might seem necessary in order to make a fair comparison between the two methods, it misses the point that on-line training can safely use a larger learning rate than batch training, and can thus train more quickly. When using a learning rate small enough for batch to converge properly, both methods will behave quite similarly, thus leading researchers to the natural conclusion that both methods are equivalent in terms of speed. Another problem is the common use of very small training sets for which the difference in training speed is less apparent.

Some researchers have even claimed that batch training is faster than on-line training. For example, in Hassoun (1995), it says "In general [the batch] update procedure converges faster than the perceptron rule, but it requires more storage" (p. 63). This reference also presents an example in which "the batch LMS rule converges to the optimal solution...in less than 100 steps. [On-line] LMS requires more learning steps, on the order of 2000 steps, to converge..." (p. 74). Each 'step,' however, was defined as one 'weight update,' and since there were 20 instances in the training set, on-line training took 20 times as many 'steps' per epoch (though the same amount of computation), thus explaining why it took 20 times as many steps overall to converge. The example continues with 'a more meaningful comparison' in which one step is a full epoch, at which point both batch and on-line have 'very similar behavior.'

The reference then concludes "Both cases (i.e. batch and on-line) show asymptotic convergence toward the optimal solution, but with a relatively faster convergence of the batch LMS rule near (the optimal solution). This is attributed to the more accurate gradient." This is a classic example of how the literature has propagated the myth that batch training is faster than on-line training. The first portion of the example is misleading, as it implies that batch training was 20 times faster than on-line training without clearly pointing out that both methods actually used the same amount of computation. Furthermore, a 20-instance training set is hardly representative of real-world problems where training time becomes an important issue. Finally, once again the learning rate for both methods was the same, which again misses the point that on-line training can use a larger learning rate (though on such a small problem this point might be less noticeable anyway).

In Demuth and Beale (1994) it says "Commonly a network which is batch updated will learn more quickly than a network which is updated based on single input and delta vectors presented sequentially" (pp. 5-7). The reference also states that "Such a batching operation often is more efficient and the results that are produced may be what is required in any case. For instance, batching all inputs in backpropagation allows the true error gradient to be calculated, which is desired" (pp. 2-13).

In Principe et al. (2000, pp. 27-28), it says "The batch algorithm is also slightly more efficient in terms of computations." In a software example, it says

Since there is more noise in on-line learning, we must decrease the step size to get smoother adaptation. We recommend using a step size 10 times smaller than the step size for batch learning. But the price paid is a longer adaptation time; the system needs more iterations to get to a predefined final error.

These statements again imply that batch training is faster than on-line training. What is not pointed out here is that their version of batch training is using the average weight change rather than the sum of weight changes (which is of course quite common), and thus is implicitly using a learning rate that is $N$ times smaller than that used by online training. In a sense, then, this statement recommends that on-line learning uses a learning rate that is " 10 times smaller than the learning rate which is $N$ times larger than the step size for batch learning," i.e. on-line cannot typically use a learning rate that is $N$ times larger than the one batch 
training uses, but may be able to use a learning rate that is $N / 10$ times as large in certain situations. (The issue of using the average vs. the sum in batch training is dealt with more in Section 3).

\subsection{Noted advantages of on-line}

Several researchers clearly state that on-line training is faster that batch training, especially in pattern recognition problems with large training sets (Bengio, 1991, 1996; Bouttou et al., 1990). In fact, Atiya and Parlos (2000) state that "It is well known that for the standard backpropagation algorithm the (on-line) update is generally faster than the batch update." Unfortunately, there are also still many who are unconvinced, though hopefully this paper will help to some degree.

Some researchers have noticed the superiority of on-line training for redundant data (Bengio, 1996; Becker \& LeCun, 1989; Bishop, 1997; Haykin, 1999). Bishop (1997) gives the example of making a training set larger by replicating it 10 times. "Every evaluation of (the gradient) then takes ten times as long, and so a batch algorithm will take ten times as long to find a given solution. By contrast, the (on-line) algorithm updates the weights after each pattern presentation, and so will be unaffected by the replication of data."

Haykin (1999) wrote, "When the training data are redundant, we find that unlike the batch mode, the (online) mode is able to take advantage of this redundancy. This is particularly so when the data set is large and highly redundant."

Bengio (1996) explained how redundancy commonly exists in large training sets.

When the training set is large, it may contain redundant information. If several gradient components-contributed from different patterns - are all pointing in a similar direction, then batch update wastes a lot of time only to obtain a more precise direction. However, the precise direction of the gradient is not necessarily the fastest direction for convergence. Furthermore, any direction within 90 degrees of the gradient also goes down the error surface (Bengio, 1996).

Some authors noted a minor advantage of on-line training to be that it requires less storage (Hassoun, 1995; Haykin, 1999; Reed \& Marks, 1999), since it does not need to store accumulated weight changes, but can apply changes to the actual weights.

Several authors also pointed out that the stochastic nature of on-line training makes it possible for it to occasionally escape from local minima (Bishop, 1997; Haykin, 1999; Hassoun, 1995; Reed \& Marks, 1999; Wasserman, 1993). For example, Hassoun (1995) says

For small constant learning rates there is a nonnegligible stochastic element in the training process that gives (on-line) backprop a quasi-annealing character in which the cumulative gradient is continuously perturbed, allowing the search to escape local minima with small and shallow basins of attraction. Thus solutions generated by (on-line) backprop are often practical ones.

While Weiss and Kulikowski (1990) did not explicitly point out the stochastic nature of on-line training, they did notice superior empirical results. They commented that "while [batch training] may have the stronger theoretical foundation, (on-line training) may yield better results and is more commonly used. In the examples we have tried, (online training) yielded superior results, in terms of smaller mean squared distances" (p. 99).

The literature offers very little empirical evidence of how often on-line training actually escapes from local minima in practice. Rather, the 'superior empirical results' of on-line training are likely due to its ability to follow the curving gradient during the course of a training epoch, and thus safely use larger learning rates than batch training and, conversely, the tendency of batch training to overshoot curves and minima.

\subsection{The open question}

Wasserman (1993) concludes a discussion on batch and on-line training by saying that "there are adherents to each method; no conclusive evidence has been presented for either alternative." We believe that this paper provides a large portion of the evidence that has been lacking.

\subsection{The many names of on-line learning}

On-line training has also been called incremental learning (Hassoun, 1995; Sarle, 2002), sample-by-sample training (Principe et al., 2000), sequential mode (Atiya \& Parlos, 2000; Bishop, 1997; Haykin, 1999), pattern-based (Bishop, 1997), pattern-by-pattern updating (Haykin, 1999), pattern-mode learning (Reed \& Marks, 1999), pattern update (Atiya \& Parlos, 2000), revision by case (as opposed to revision by epoch) (Weiss \& Kulikowski, 1990), and revision by pattern (Weiss \& Kulikowski, 1990). However, the most common name appears to be on-line training (Haykin, 1999; Principe et al., 2000; Reed \& Marks, 1999; Wasserman, 1993).

The name 'on-line' is not quite accurate, because training is typically done with the system off-line even when using so-called on-line training, which may explain why so many attempts have been made to rename it. As Reed and Marks (1999) explain, “The label 'on-line learning' may be confusing because it implies that learning may occur in the field during normal operation and that it is not necessary to take the system off-line for training. But on-line learning, like batch-mode learning, is normally done off-line during a separate training phase with controlled data sets." However, since the purpose of this paper is to call attention to 
the superiority of an existing algorithm rather than to introduce a new one, we use the name on-line (with apologies) in order to avoid confusion resulting from a less familiar name.

\section{Gradient descent learning}

There are many different algorithms that use a gradient descent approach to learning (Hassoun, 1995), such as the perceptron learning rule (Rosenblatt, 1962), competitive learning (Rumelhart \& McClelland, 1986), reinforcement learning (Barto, 1992), and self-organizing maps (Kohonen, 1982).

Gradient descent learning attempts to find a point $\mathbf{W}$ in some parameter space (e.g. neural network weight space) that minimizes an error (or 'loss') function $L(\mathbf{W})$. The weight space and error function define an error surface. If there were only two weights, an error surface could be visualized as a hilly landscape, as illustrated in Fig. 1 . The $(x, y)$ coordinates represent values of the two weights, and the height represents the error of the training set when evaluated at each weight setting. In practical problems there can be many thousands of weights, making the error surface difficult to visualize, but this analogy helps in understanding what is going on with gradient descent learning.

The gradient descent algorithm starts at some (usually random) point in the weight space and moves 'downhill' until a minimum in the error surface is found, moving in the direction of steepest descent at each point. A true gradient descent algorithm would continuously move in the (negative) direction of the instantaneous gradient, somewhat like a drop of water trickling downhill.

However, in implementations of gradient descent algorithms such as error backpropagation, gradient descent

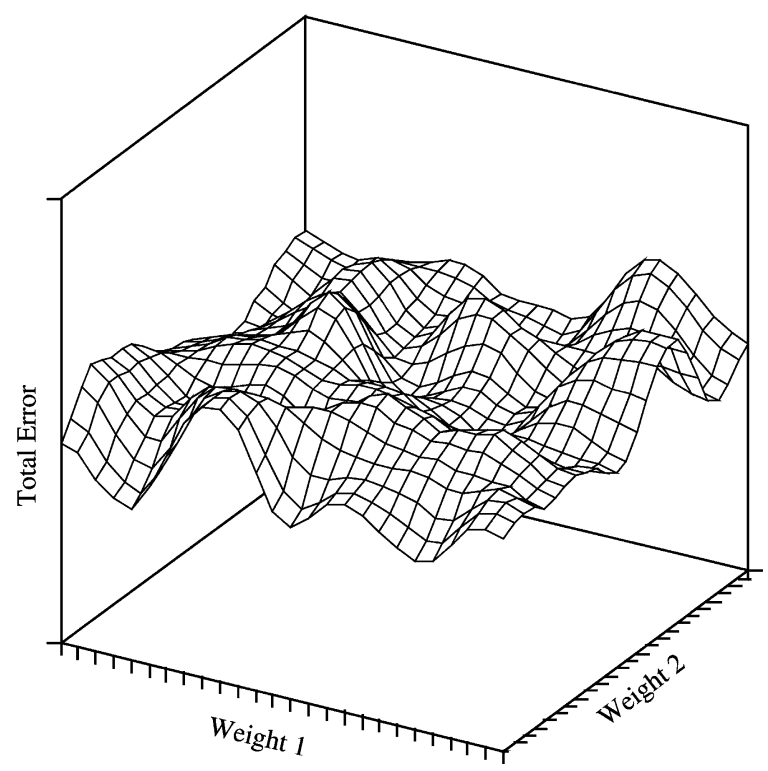

Fig. 1. Illustration of an error surface. Two weights define the $x-y$ plane, and the height indicates the error at each point in weight space.

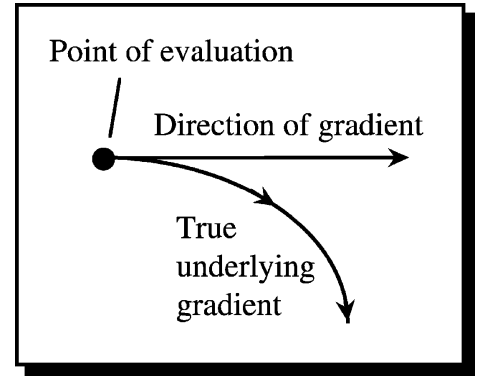

Fig. 2. The gradient is measured at a point, and steps are taken in that direction, even though the true underlying gradient may curve continuously.

is approximated by taking small but finite steps in the direction of steepest descent, i.e. $-r \cdot \partial E / \partial w$, where $r$ is the learning rate. The gradient is only valid at the point at which it was measured. As soon as the weights just start to change in the direction of the gradient at the measured point, the true underlying gradient itself will often start to change, as shown in Fig. 2. Therefore, implementations of so-called gradient descent algorithms are only truly gradient descent as their learning rates approach zero, at which point training times approach infinity. Thus, such algorithms must approximate gradient descent by taking discrete steps in weight space, moving in a straight line in the direction of the gradient as measured at a particular point, even though the gradient may curve during the course of that step.

\subsection{The effect of learning rates on training time and generalization accuracy}

The size of the learning rate $r$ is critical to the success and efficiency of the learning algorithm. A learning rate that is too large will cause the algorithm to take huge steps in weight space, stepping over valleys and missing curves in the error landscape, and thus overshooting good paths and solutions. This makes generalization accuracy worse and also takes longer to train, because it is continually overshooting its objective and 'unlearning' what it has learned. On the other hand, a learning rate that is too small will waste computation, using many steps to move to essentially the same point in weight space that could have been reached in fewer steps.

Fig. 3 shows a summary of phoneme recognition experiments reported by the authors in (Wilson \& Martinez, 2001). The horizontal axis shows a variety of learning rates that were used. For each learning rate, the maximum phoneme recognition accuracy is plotted (using the vertical scale on the right), along with the number of training epochs required to reach that level of accuracy (using the vertical scale on the left).

As can be seen from the figure, the generalization accuracy gets better as the learning rate gets smaller, and the training time also improves dramatically. At a learning rate of about 0.01 , the accuracy and training time are both close to their 


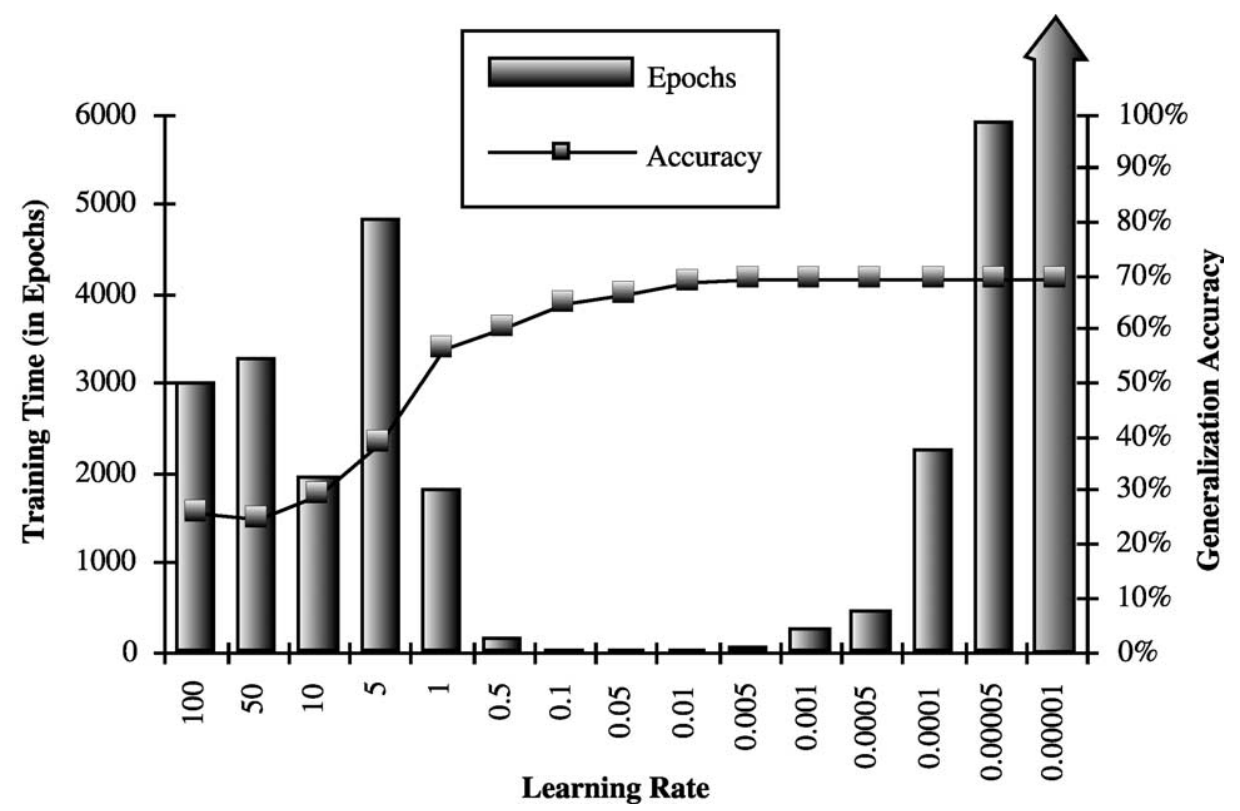

Fig. 3. Training time (in epochs) and maximum hold-out set phoneme generalization accuracy for each learning rate, on a digit speech recognition task. The bars indicate the number of epochs needed to reach the maximum generalization accuracy, and the line indicates what the maximum accuracy was for each learning rate.

maximums. It often is possible to get slightly higher accuracy by using an even smaller learning rate than the 'fastest' one, as is the case here, where accuracy improves by using a learning rate of 0.001 , at the expense of increased training time. Beyond that, however, smaller learning rates require linearly more training time with no significant improvement in accuracy (Wilson \& Martinez, 2001).

\subsection{Deterministic and stochastic optimization}

Let $\mathbf{W}$ be the multidimensional vector of weights (or other parameters) being trained via gradient descent. Let $L(\mathbf{W})$ be the error (or loss) over the training set evaluated at the point $\mathbf{W}$ in the weight space. Then the optimization problem is to find a point $\mathbf{W}$ that minimizes $L(\mathbf{W})$.

In gradient descent learning, the (negative) gradient of the error with respect to the weights is used to choose the direction in which to move the weights in order to reduce the error, until a (local) minimum is found.

Let $T$ be a training set consisting of $N$ instances, $\left\{x_{1}, x_{2} \ldots, x_{N}\right\}$. These instances can be randomly reordered after each epoch. In batch training the order of presentation is irrelevant, so we are free to assume that the order of presentation is the same for batch and on-line training during each epoch $t$.

Let $\mathbf{G}(\mathbf{W})$ be the gradient of the error over $T$ with respect to the current weight setting $\mathbf{W}$, i.e. $\mathbf{G}(\mathbf{W})=-\partial L / \partial \mathbf{W}$. Let $g\left(x_{i}, \mathbf{W}\right)$ be the (negative) 'local' (or 'per-instance') gradient for a particular instance $x_{i}$ in the training set, evaluated at W. Then

$\mathbf{G}(\mathbf{W})=\sum_{i=1}^{N} g\left(x_{i}, \mathbf{W}\right)=-\partial L / \partial \mathbf{W}$ i.e. the gradient over the entire training set is equal to the sum of the local gradients for each instance in the training set. Similarly, let $\overline{\mathbf{G}}(\mathbf{W})$ be the average of the local gradients of all of the instances, i.e. $\overline{\mathbf{G}}(\mathbf{W})=\mathbf{G}(\mathbf{W}) / N$. Both the sum and the average point in the direction of the gradient, and this section uses each where appropriate to simplify the notation. (Further clarification on this is given below.)

Let $\mathbf{W}_{t, i}$ be the weights after processing $t$ epochs of the entire training set plus $i$ instances of the current epoch. $\mathbf{W}_{0,0}$ is the initial weight setting, and $\mathbf{W}_{t+1,0}=\mathbf{W}_{t, N}$, so that each epoch begins where the previous one leaves off.

\subsubsection{Deterministic optimization}

In deterministic optimization, such as batch training for gradient descent, the gradient $\mathbf{G}(\mathbf{W})$ is used to determine the direction of the next step of the optimization. The batch gradient descent algorithm thus has the form

$\mathbf{W}_{t+1,0}=\mathbf{W}_{t, 0}+r \cdot \mathbf{G}\left(\mathbf{W}_{t, 0}\right)=\mathbf{W}_{t, 0}+r \cdot \sum_{i=1}^{N} g\left(x_{i}, \mathbf{W}_{t, 0}\right)$

where $r$ is the learning rate.

Eq. (2) can be rewritten recursively as:

$\mathbf{W}_{t, i+1}=\mathbf{W}_{t, i}+r \cdot \mathbf{g}\left(x_{i}, \mathbf{W}_{t, 0}\right)$, for $1 \leq i \leq N$

with $\mathbf{W}_{t, 0}=\mathbf{W}_{t-1, N}$ for $t>0$, and an initial weight setting of $\mathbf{W}_{0,0}$.

For batch training, it is typical to think of 'accumulating' weight changes for $i=1 \ldots N$, and then to 'apply' them after they are all accumulated. Readers are encouraged to examine the above recursive equation carefully to satisfy themselves that this is equivalent to such a method, i.e. $\mathbf{W}_{t, 0}$ can be thought of as the 'real' weights after $t$ epochs of 
training, and as can be seen from the equation, $\mathbf{W}_{t, 0}$ is what is always used to calculate the local gradient for each instance. $\mathbf{W}_{t, 1}$ through $\mathbf{W}_{t, N}$ are thus not used in the equation except to accumulate the weight changes.

\subsubsection{Stochastic optimization}

In stochastic optimization (Kiefer \& Wolfowitz, 1952; Robbins \& Monro, 1951; Spall, 1999), on the other hand, an estimate $\mathbf{Y}(\mathbf{W})$ of the gradient is used at each step. The Robbins-Monro Stochastic Approximation (RMSA) algorithm (Robbins \& Monro, 1951) has the form

$\mathbf{W}_{t, i+1}=\mathbf{W}_{t, i}+r_{k} \cdot \mathbf{Y}\left(\mathbf{W}_{t, i}\right)$

where $r_{k}$ is the learning rate after processing $k$ instances, i.e. $k=t N+i$.

Convergence can often be guaranteed if $r_{k}$ decays according to certain constraints. ${ }^{2}$ Even when a constant step size is used, a partial convergence theory is often possible (Spall, 1999) to within some error of the local minimum. In our experiments, we have seen slight improvements in solutions obtained by using a decaying learning rate, so in practice this is often useful. However, in order to more directly compare batch and on-line training, a constant learning rate will be used in the remainder of this paper.

It would of course be preferable to use the deterministic gradient $\overline{\mathbf{G}}(\mathbf{W})$ instead of the stochastic estimate $\mathbf{Y}(\mathbf{W})$ at each step in this optimization, if these two values took the same amount of time to compute. However, $\overline{\mathbf{G}}(\mathbf{W})$ takes $N$ times as long to compute as $\mathbf{Y}(\mathbf{W})$. As will be shown below, taking $N$ stochastic steps $\mathbf{Y}(\mathbf{W})$ per epoch actually follows the gradient more closely and is thus able to make much more progress than taking a single step in the direction of $\overline{\mathbf{G}}(\mathbf{W})$.

For on-line training, we use $\mathbf{Y}\left(\mathbf{W}_{t, i}\right)=\mathbf{g}\left(x_{i+1}, \mathbf{W}_{t, i}\right)$, i.e. the per-instance gradient for each instance is used as an estimate of the true gradient over the entire training set, yielding an update equation of:

$\mathbf{W}_{t, i+1}=\mathbf{W}_{t, i}+r \cdot \mathbf{g}\left(x_{i+1}, \mathbf{W}_{t, i}\right)$

While it is true that $\mathbf{Y}(\mathbf{W})$ is not equal to $\overline{\mathbf{G}}(\mathbf{W})$, the expected value of $\mathbf{Y}$ does equal $\overline{\mathbf{G}}$ (under fairly modest regularity conditions) (Glasserman, 1991; Spall, 1999). This is an extremely important point, and so bears repeating:

The expected value of the per-instance gradient used by on-line training is the true gradient at that point, i.e. $E\left(\mathbf{g}\left(x_{i}, \mathbf{W}\right)\right)=\overline{\mathbf{G}}(\mathbf{W})$.

\footnotetext{
${ }^{2}$ Convergence under RMSA is guaranteed when (a) $r_{t, i}>0$; (b) $\sum_{k-1}^{\infty} r_{k}=\infty$; and (c) $\sum_{k-1}^{\infty} r_{k}^{2}<\infty$; e.g. $r_{k}=a /(k+1)$ is a common choice, though not necessarily optimal for finite training sets (Robbins and Monro, 1951; Spall, 1999).
}

To see that this is so, observe that $P\left(x_{i}\right)=1 / N$, since the instances are presented in random order with equal probability. Therefore,

$$
\begin{aligned}
& E\left(\mathbf{g}\left(x_{i}, \mathbf{W}\right)\right)=\sum_{i=1}^{N} P\left(x_{i}\right) \cdot \mathbf{g}\left(x_{i}, \mathbf{W}\right)=\sum_{i=1}^{N} \frac{1}{N} \cdot \mathbf{g}\left(x_{i}, \mathbf{W}\right) \\
& =\frac{1}{N} \sum_{i=1}^{N} \mathbf{g}\left(x_{i}, \mathbf{W}\right)=\frac{\mathbf{G}(\mathbf{W})}{N}=\overline{\mathbf{G}}(\mathbf{W})
\end{aligned}
$$

\subsection{Why batch training is slower than on-line training}

Given the above groundwork, it is now possible to show why batch training can be expected to be slower than on-line training, especially as the size of the training set grows large.

\subsubsection{Similarity of batch and on-line training}

To show more clearly just how similar batch and on-line training are, the batch and on-line training update equations (as applied for each instance in the training set) are repeated below for comparison:

Batch : $\mathbf{W}_{t, i+1}=\mathbf{W}_{t, i}+r \cdot \mathbf{g}\left(x_{i}, \mathbf{W}_{t, 0}\right)$

On-line : $\mathbf{W}_{t, i+1}=\mathbf{W}_{t, i}+r \cdot \mathbf{g}\left(x_{i+1}, \mathbf{W}_{t, i}\right)$

From these update equations, it can be seen that the only difference between the two is that on-line training uses the weights at the current point in weight space, while batch uses the weights at the original point in weight space (for each epoch), when determining how to update the weights for each instance.

As the learning rate $r$ approaches 0, less change in the weights is made per epoch, which means that $\mathbf{W}_{t, i} \rightarrow \mathbf{W}_{t, 0}$. Thus batch and on-line training become identical in the limit as $r \rightarrow 0$. In practice, if the learning rate is small enough for batch training to follow the gradient closely, on-line learning will usually follow essentially the same path to the same minimum.

Some authors define batch training as using the average of the weight changes over an epoch of $N$ instances (Principe et al., 2000; Wasserman, 1993) rather than the sum. These are of course equivalent if one uses a learning rate of $r / N$ in the former case and $r$ in the latter. However, as can be seen from the above equations, using the average for batch training (i.e. $r / N$ ) would result in batch training being $N$ times slower than on-line training in the limit as $r \rightarrow 0$. We therefore use the sum in batch training in order to make the comparison fair.

\subsubsection{Example of basic difference}

Fig. 4(a) shows an example of several individual weight changes (represented as vectors in 2-space) collected during 
an epoch of batch training. The long dashed line represents the sum of these individual vectors and shows the direction of the true (training set error) gradient.

This sum can also be graphically represented by placing the same vectors end-to-end, as in Fig. 4(b). When looked at in this way, batch training can be thought of as 'updating' its weights after each instance, just as in on-line training, but computing the local gradient at each step with respect to the original point in weight space, as reflected in the above equations. In other words, as it travels through weight space during each epoch, it evaluates each instance at the original point $\left(\mathbf{W}_{t, 0}\right)$ to decide where it should go from its current point $\left(\mathbf{W}_{t, i}\right)$, even though the gradient may have changed considerably between those two points.

On-line training, on the other hand, always uses the current point in weight space $\mathbf{W}_{t, i}$ to compute the local gradient, and then moves from there. This allows it to follow curves in the error landscape. Fig. 4(c) illustrates the path of on-line training for the same instances as in Fig. 4(a) and (b). It varies from the true gradient at each point by a similar amount and at a similar angle to the true gradient, but since it can use the gradient at the current point instead of the original point, it follows curves and makes much more progress during the epoch.

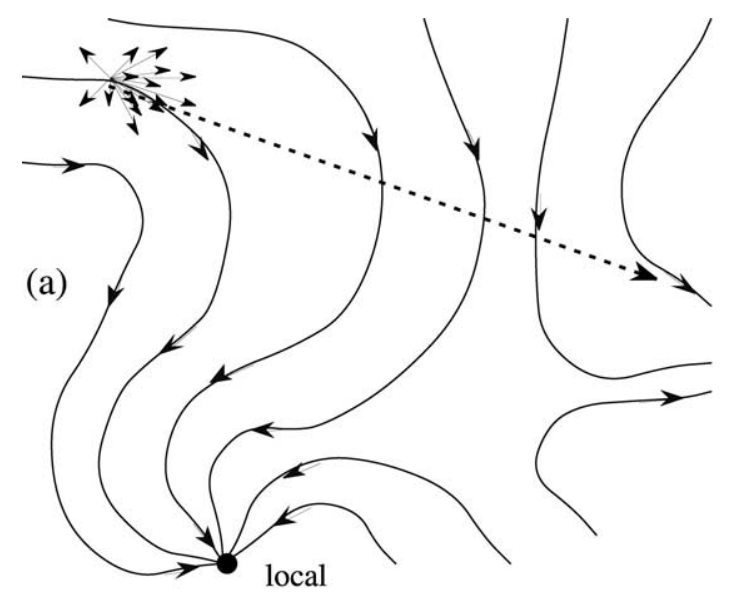

minimum
In contrast, observe how after the first few steps, batch training is heading in a general direction that is nearly perpendicular to the current true gradient. This illustrates the point that while batch training computes the direction of the true gradient, this direction is only valid at the starting point. As the weights actually start to move in that direction, the direction of the true gradient at the original point is being used as an estimate of what the gradient is along the rest of the path. The further along the path the weights get, the less accurate this estimate becomes.

In on-line training, the path through weight space will fluctuate around the gradient direction (Principe et al., 2000) as it curves its way to the minimum, as illustrated earlier in Fig. 4(c). Batch training, on the other hand, can be thought of as fluctuating around the fixed gradient calculated at the original point, as shown by the jagged path taken in Fig. 4(b). Alternatively, it can be thought of as diverging from the true gradient in a straight line, as illustrated by the dashed line in the same figure.

When on-line training makes 'mistakes' by overshooting a minimum, missing a curve, or by moving in a wrong direction due to its noisy estimate of the gradient, it can start to make corrections immediately with the very next instance. This is because each of its stochastic updates is

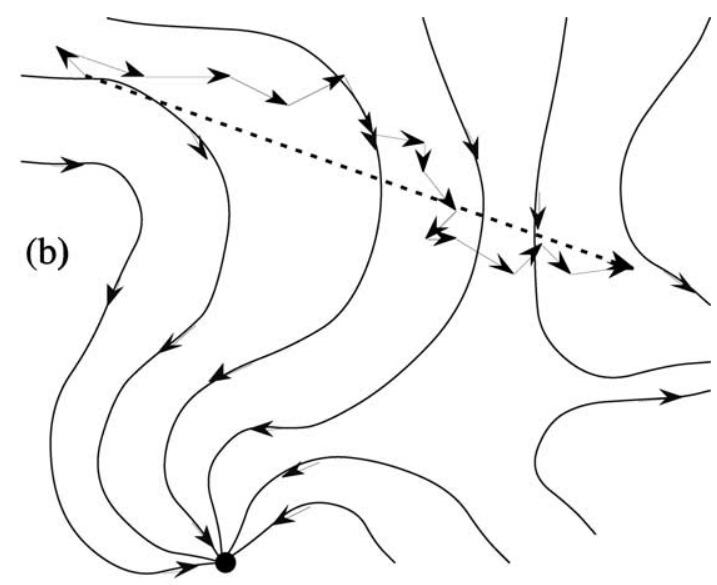

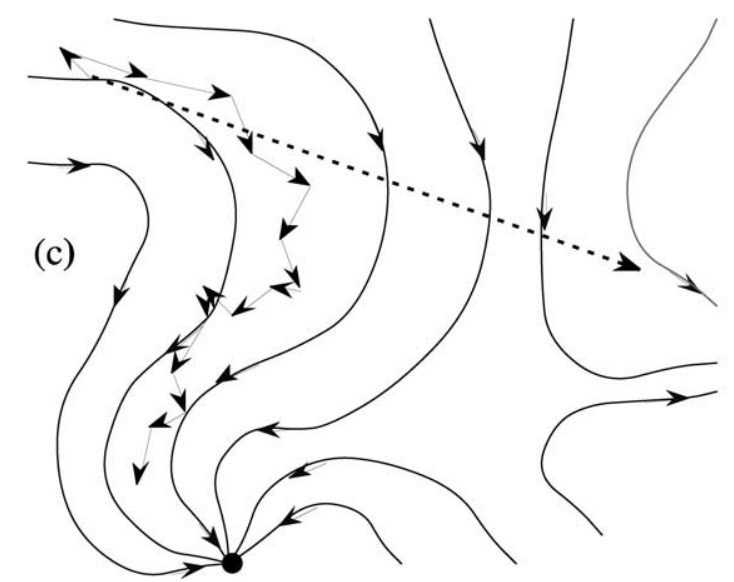

Fig. 4. Example of changes in weight space. The directed curves indicate the underlying true gradient of the error surface. (a) Batch training. Several weight change vectors and their sum. (b) Batch training with weight change vectors placed end-to-end. Note that batch training ignores curves and overshoots a 'valley,' thus requiring a smaller learning rate. (c) On-line training. The local gradient influences the direction of each weight change vector, allowing it to follow curves. 
based on the current gradient at the new point. When batch training makes mistakes by overshooting a minimum or failing to follow a curve, it just keeps on going straight until the beginning of the next epoch.

\subsubsection{Neither uses the 'true' gradient: both estimate}

Thus, both batch and on-line training are using approximations of the true gradient as they move through weight space. On-line training uses a rough estimate of the current gradient at each of $N$ steps during an epoch, while batch training carefully estimates the gradient at the starting point, but then ignores any changes in the gradient as it takes one large step (equivalent to $N$ small average-sized straight steps) in that direction. Of the two, batch actually tends to use the less accurate estimate in practice, especially as the learning rate and/or the size of the training set increases, since both of these factors increase the distance of the ending point (where the weights move to) from the initial point in weight space (where the gradient was calculated).

Even if we knew the optimal size of a step to take in the direction of the gradient at the current point (which we do not), we usually would not be at the local minimum, since the gradient will curve on the way to the minimum. Instead, we would only be in a position to take a new step in a somewhat orthogonal direction, as is done in the conjugate gradient training method (Møller, 1993).

Of course, when using a finite training set, even the true gradient is in practice the gradient of the training set error with respect to the weights, not the gradient of the true underlying function we are trying to approximate, though this is true for both on-line and batch training.

\subsubsection{Brownian motion}

Another way to think of on-line training is in terms of Brownian motion. When a particle exhibits Brownian motion, it moves in random directions at each point, but is biased towards moving in the direction of an underlying current. Similarly, as on-line training progresses, each weight change can be in any direction, but the probability distribution is skewed towards the true gradient.

The direction of movement at each point can thus be looked at as a combination of a noise vector pointing in a random direction and a gradient vector pointing in the direction of the true gradient. In the long run, the law of averages will tend to cancel out much of the noise and push the weights in the direction of the true gradient. Under this interpretation, the main difference between batch and online training is that while both will have similar noise vectors, batch training must use a fixed gradient vector in all of its weight accumulations for an entire epoch, while online training is allowed to use the current gradient vector for each of its weight updates.

Put differently, the expected value of the weight change for each instance is $r \cdot \overline{\mathbf{G}}\left(\mathbf{W}_{t, i}\right)$ for on-line training and $r \cdot \overline{\mathbf{G}}\left(\mathbf{W}_{t, 0}\right)$ for batch training. After every $N$ instances (i.e. one pass through the training set), the expected value of the weight change for batch training suddenly changes to point in the direction of the gradient at the end of the previous epoch, and then remains fixed until the end of the next epoch. In contrast, the expected value of the weight change for on-line training is continuously pointing in the direction of the gradient at the current point in weight space. This allows it to follow curves in the gradient throughout an epoch.

\subsubsection{Large training sets}

As the size of the training set grows, the accumulated weight changes for batch training become large. For example, consider a training set with 20,000 instances. If a learning rate of 0.1 is used, and the average gradient is on the order of \pm 0.1 for each weight per instance, then the total accumulated weight change will be on the order of $\pm(0.1 \times$ $0.1 \times 20,000)= \pm 200$. Considering that weights usually begin with values well within the range of \pm 1 , this magnitude of weight change is very unreasonable and will result in wild oscillations across the weight space, and extreme overshooting of not only the local minima but the entire area of reasonable solutions.

In networks such as multilayer perceptrons, such large weight changes will also cause saturation of nonlinear (e.g. sigmoidal) units, making further progress difficult. In terms of the hyperplanes or other hypersurfaces constructed by neural network architectures, such overshooting often equates to putting all of the data on one side of the 'decision' surfaces with nothing on the other side, resulting in random or nearly random behavior. Thus, a reduction in the size of the learning rate is required for batch training to be stable as the size of the training set grows. (This is often done implicitly by using the average weight change instead of the sum, effectively dividing the learning rate by $N$ ).

On-line learning, on the other hand, applies weight changes as soon as they are computed, so it can handle any size of training set without requiring a smaller learning rate. In the above example, each weight change will be on the order of $\pm 0.1 \times 0.1= \pm 0.01$, even with a learning rate of 0.1 . If we consider a 'safe' step size for this example to be on the order of \pm 0.01 , then batch might need to use a learning rate as small as $r=0.1 / N=0.00005$ in order to take steps of that size.

Since some of the individual weight changes cancel each other out, the learning rate does not usually need to be a full $N$ times smaller for batch than for on-line, but it does usually need to be much smaller. In the above example $r$ would perhaps need to be 200 times smaller, or $r=$ $0.1 / 200=0.0005$. This in turn means that batch training will require many more (e.g. 200 times as many) iterations through the training data when compared to on-line training.

In our experiments it was common to have to reduce the learning rate by a factor of around $\sqrt{N}$ to get batch training to train with the same stability as on-line training on a training set of $N$ instances. This is only a rough estimate, and the precise relationship between training set size and 
reduction in learning rate required for batch training is highly task-dependent. (Of course, once we know that online training is significantly faster than batch and at least as accurate, it may be less important to know exactly how much faster it is.)

As the training set gets larger, on-line training can make more progress within each epoch, while batch training must make the learning rate smaller (either explicitly or by using the average weight change) so as not to go too far in a straight line. With a sufficiently large training set, on-line training will converge in less than one epoch before batch training takes its first step. In the limit, with an infinite training set, batch training would never take a step while on-line training would converge as usual.

\subsection{Choosing a learning rate}

One concern that might arise is that even if on-line training can use a larger learning rate than batch training, there is in general no way of knowing what size of learning rate to use for a particular task, and so there is no guarantee of finding the larger learning rate that would allow on-line training to learn faster. However, both on-line and batch training share this problem, so this cannot be seen as a comparative disadvantage to on-line training. In fact, since on-line training can use a larger learning rate, the search for an appropriate learning rate is easier with on-line training, since the entire range of learning rates safe for use in batch training are also available for on-line training, as well as an additional range of larger rates. Furthermore, since these larger learning rates allow for faster training, the process of searching for learning rates itself can be sped up, because larger learning rates can be quickly checked and smaller ones can then be more quickly ruled out as they fail to improve upon earlier results.

With on-line training, 'the weight vector never settles to a stable value' (Reed \& Marks, 1999), because the local gradients are often non-zero even when the true gradient is zero. The learning rate is therefore sometimes decayed over time (Bishop, 1997). In our experiments we held the learning rates constant throughout training so that comparisons of training time would be more meaningful, but it might be advantageous to reduce the learning rate after approximate convergence has been reached (Reed \& Marks, 1999).

\subsection{Momentum}

The experiments in this paper have not used the momentum term. When the momentum term is used, the process is no longer gradient descent anyway, though it has in some cases sped up the learning process. Given a momentum term of $\alpha$, with $0 \leq \alpha \leq 1$, the effective learning rate is $r_{\alpha}=r /(1-\alpha)$ when weight changes are in approximately the same direction. Momentum can thus have an amplification effect on the learning rate, which would tend to hurt batch training more than on-line training. Momentum can also help to 'cancel out' steps taken in somewhat opposite directions, as when stepping back and forth across a valley. In such cases the unproductive elements tend to cancel out, while the common portions tend to add to each other.

We ran just a few experiments using momentum, and from these it appeared that on-line retained the same advantage over batch in terms of speed and smoothness of learning, and that indeed the learning rate was amplified slightly. On the Australian task, for example, using a momentum of $\alpha=0.9$ and a learning rate of $r=0.001$ resulted in training similar to using a learning rate between $r=0.001$ and $r=0.01$ for both batch and on-line training.

\subsection{Batch/on-line hybrid}

Another approach we tried was to do on-line training for the first few epochs to get the weights into a reasonable range and follow this by batch training. Although this helps to some degree over batch training, empirical results and analytical examination show that this approach still requires a smaller learning rate and is thus slower than on-line training, with no demonstrated gain in accuracy.

Basically, such a hybrid approach is the same as on-line training until the epoch at which batch training begins, and then must use the same small learning rate as when batch training is used alone in order to do stable learning.

\section{Empirical results}

From the discussions in preceding sections, we hypothesized that on-line training is usually faster than batch training, especially for large training sets, because it can safely use a larger learning rate, due to its ability to follow curves in the error surface. However, how much faster online is depends on both the size of the training set and the characteristics of the particular application. As discussed above, as the training set gets larger, we expect the speedup of on-line over batch to increase.

The smoothness of the underlying error surface also influences training speed. A smoother surface usually allows a larger learning rate which typically allows faster convergence. However, since both on-line and batch training can use a larger learning rate on a smoother surface, smoothness may not have as much effect as other factors on speedup.

The amount of speedup of on-line over batch also depends on how much cooperation there is among the 'local gradients' of the individual training instances. If the individual weight changes for all $N$ instances in the training set happen to be in the direction of the true (current) gradient, then on-line training would be able to progress $N$ times as fast as the corresponding batch algorithm, since it 
could make the same progress per instances as batch would per epoch.

On the other hand, the individual on-line training steps could be so contradictory that the total amount of progress made in an entire epoch is less than or equal to the size of a typical single step in on-line training. This case can be thought of as $N-1$ steps canceling each other out, leaving one final step (or partial step) of actual progress in the direction of the true gradient. Alternatively, it can be thought of as $N$ steps, each of which moves only $1 / N$ of a full step or less in the right direction (on average). Either way, the additional steps taken by on-line training in such situations are 'wasted' (though no more so than the similar accumulations made by batch) so training is reduced to being no faster than batch training.

In more realistic cases, we expect there to be some contradiction between the weight changes for individual training instances because they each favor their own output values, but also some cooperation among many instances in the direction of the true gradient. Determining the amount of such cooperation analytically is very difficult for real-world tasks, and it varies widely depending on the characteristics of each task. Therefore, empirical results are needed to explore the question of how much faster on-line is than batch training in practice.

Two sets of experiments were run, one using a collection of classification tasks with various sizes of training sets, and another using a large training set for a speech recognition task. In both cases various learning rates were used for online and batch training. In the speech recognition task, minibatch training was also used with various update frequencies ('batch sizes') to further explore the relationship between batch size, learning rate, and training speed.

The experiments in this paper use multilayer perceptrons (MLP) trained using the error backpropagation (BP) algorithm (Rumelhart \& McClelland, 1986).

\subsection{Machine learning database experiments}

One problem with earlier comparisons between batch and on-line training is that they have used a single learning rate to compare the two, ignoring the fact that on-line training can often use a larger learning rate than batch training. Another problem is that they often used artificial and/or 'toy' (very small, simple) problems for comparison, thus missing differences that occur in larger problems.

In order to test the hypothesis that on-line training trains faster than batch training, experiments were run on 26 classification tasks from the UCI Repository of Machine Learning Databases (Blake \& Merz, 1998). For each task, $60 \%$ of the data was used for training, $20 \%$ for a hold-out set, and the remaining $20 \%$ as a final test set. The hold-out set was used to test the generalization accuracy after each epoch of training, and the final test set was used to measure accuracy at the epoch where generalization was the highest on the hold-out set.

Each task was trained using both on-line and batch training methods, and in each case learning rates of 0.1 , $0.01,0.001$, and 0.0001 were used. Ten trials were run for each of the four learning rates for both methods on each of the 26 tasks, for a total of $10 \times 4 \times 2 \times 26=2080$ runs.

Each run used error backpropagation to train a multilayer perceptron. For a classification task with $X$ inputs and $Z$ output classes, the architecture of the multilayer perceptron was set up to have $X$ input nodes, one layer of $X \times 2$ hidden nodes, and $Z$ output nodes. During training the target value of the output node corresponding to the target output class was set to 1 , and the target value of the other nodes was set to 0. During testing, an instance was considered to be classified correctly if the target output class had the highest activation of any of the output nodes. The generalization accuracy on the hold-out and test set was defined the percentage of instances correctly classified in the hold-out or test set, respectively.

The neural networks in each experiment were initialized with small random weights, and the order of presentation of instances was randomized such that it was different for each epoch. The random number generator was seeded with a number that was determined by which of the ten trials the experiment was in. Thus, each of the ten trials started with different random initial weights, but the batch and on-line methods for each experiment always used the same random initial weights for each of their corresponding trials. Similarly, each of the ten trials used the same random initial weights for all four learning rates, and used the same order of presentation of instances for both on-line and batch training as well as for the four different learning rates.

Each neural network was trained for 1000 epochs for learning rates 0.1 and $0.01 ; 5000$ epochs for 0.001 ; and 10,000 epochs for learning rate 0.0001 . Each neural network was then tested on the hold-out set and the results for each epoch over the ten trials were averaged. A few tasks had generalization accuracy that was still rising after the number of epochs listed above. In those cases, additional training epochs were used in order to determine how long batch and on-line training took to train to a maximum accuracy in each case.

We first show a representative example of the behavior of batch and on-line training in detail, and then discuss the average results over all 26 tasks.

\subsubsection{Example: Mushroom}

The Mushroom dataset has 3386 instances in its training set, and generalization accuracy after each epoch is plotted in Fig. 5 for the four learning rates. As shown in Fig. 5(a), for a learning rate of 0.1 , on-line training fluctuates on its way to an asymptote at about 98.5\% accuracy, which it reaches after about 800 epochs, while batch training remains completely random. In Fig. 5 (b), a learning rate of 0.01 allows on-line training to 
(a)

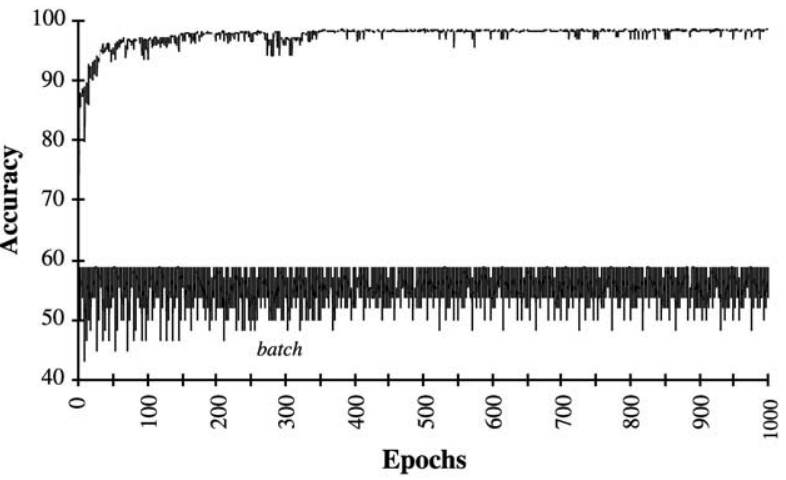

(c) $r=0.001 \quad$ Mushroom

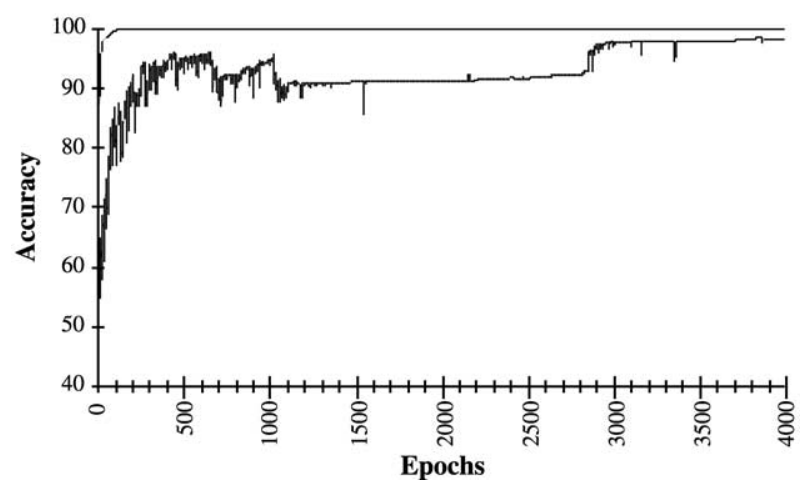

(b) $r=0.01$

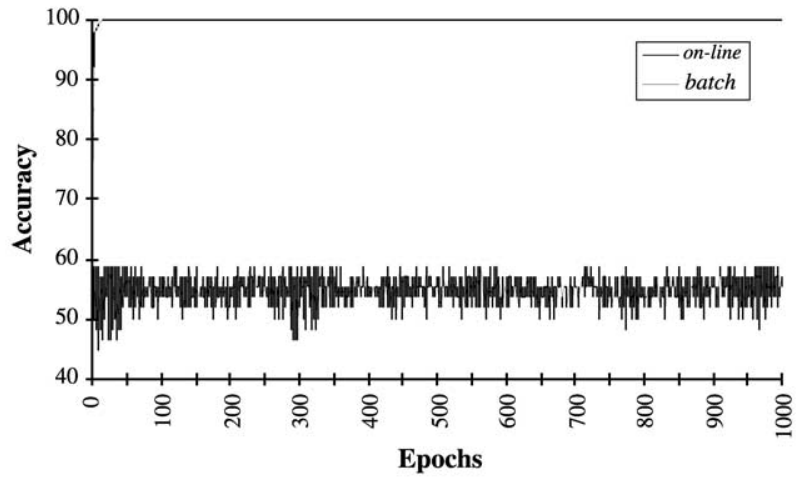

(d) $r=0.0001$

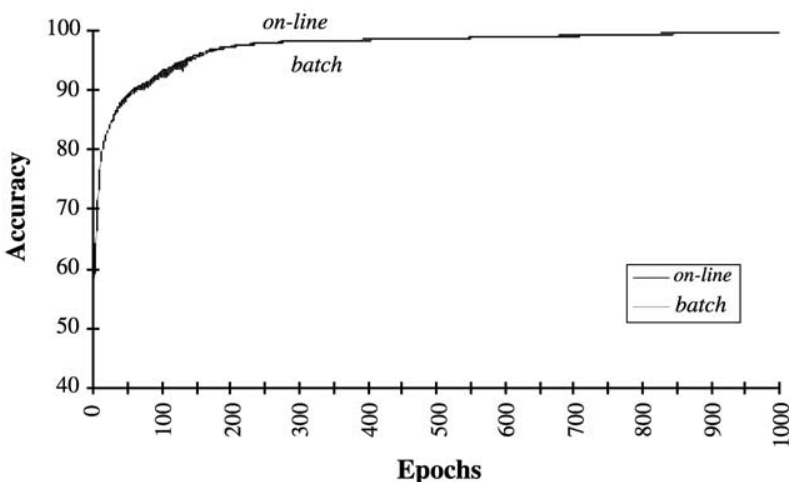

Fig. 5. Generalization accuracy of on-line and batch training on the Mushroom task, using learning rates of (a) 0.1, (b) 0.01 , (c) 0.001, and (d) 0.0001.

smoothly approach $100 \%$ generalization accuracy after only 20 epochs, while batch training continues to remain random. In Fig. 5(c), batch training finally makes some progress with a learning rate of 0.001 , but still achieves only a $98.5 \%$ accuracy after 3800 epochs. Finally, with a learning rate of 0.0001 , batch training follows on-line training quite closely, as shown in Fig. 5(d), dipping only slightly a few times, and both reach $100 \%$ accuracy after about 1800 epochs.

In this example, batch training required 1800 epochs to reach $100 \%$ using its best learning rate of 0.0001 , while online training was able to do so in 20 epochs using a learning rate of 0.01 .

In this case it is easy to see that if someone used a learning rate small enough for batch training to convergei.e. 0.0001 - and compared batch and on-line training using this same learning rate, they might conclude that there is no significant difference between the two. But since on-line learning can use a larger learning rate, it is in fact able to learn 90 times as fast in this case.

It is common for people to use the average weight change in batch training instead of the sum. With a learning rate of $r \times N=0.0001 \times 3386=0.3386$, taking the average would yield the exact same results as using the sum with a learning rate of 0.0001 . This agrees with the rule of thumb of Principe et al. (2000), as mentioned in Section 2.1, that when using the average weight change, batch training can use a learning rate that is an order of magnitude larger than on-line training (0.3386 vs. 0.01 , in this case, to achieve smooth learning). Regardless of whether the sum (with learning rate 0.0001 ) or the average (with learning rate of 0.3386) is used, however, the result is the same: batch training requires 90 times as many iterations as on-line training when both are allowed to use their 'best' learning rate.

In the remainder of this paper, the sum is used, because using the sum has the property that as the learning rate becomes small, batch and on-line training become equivalent.

\subsubsection{Overall results}

Fig. 6 shows the average accuracy of all 26 tasks after each epoch of training for the same learning rates. For learning rates of $0.1,0.01,0.001$ and 0.0001 , batch training is on average about $30,12.5,4$ and $0.8 \%$ lower than on-line training, respectively, at any particular epoch. This shows again that the smaller the learning rate is, the more closely batch and on-line training behave. It also demonstrates that on-line training generally learns more quickly (as well as more 'safely') with larger learning rates. The small difference observed in Fig. 6(d) is almost entirely due to the Letter Recognition task which has 12,000 instances in 

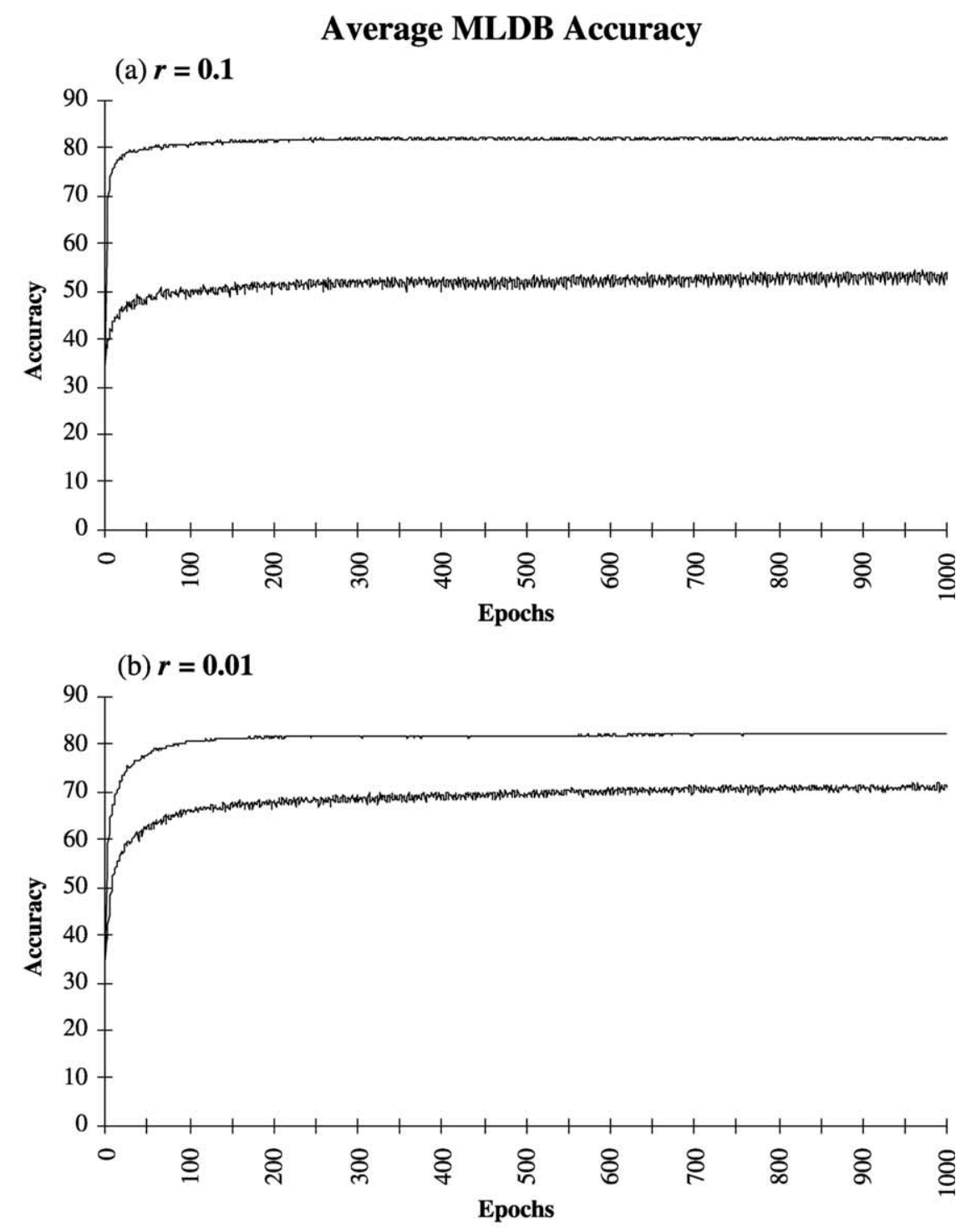

(c) $\boldsymbol{r}=\mathbf{0 . 0 0 1}$

Average

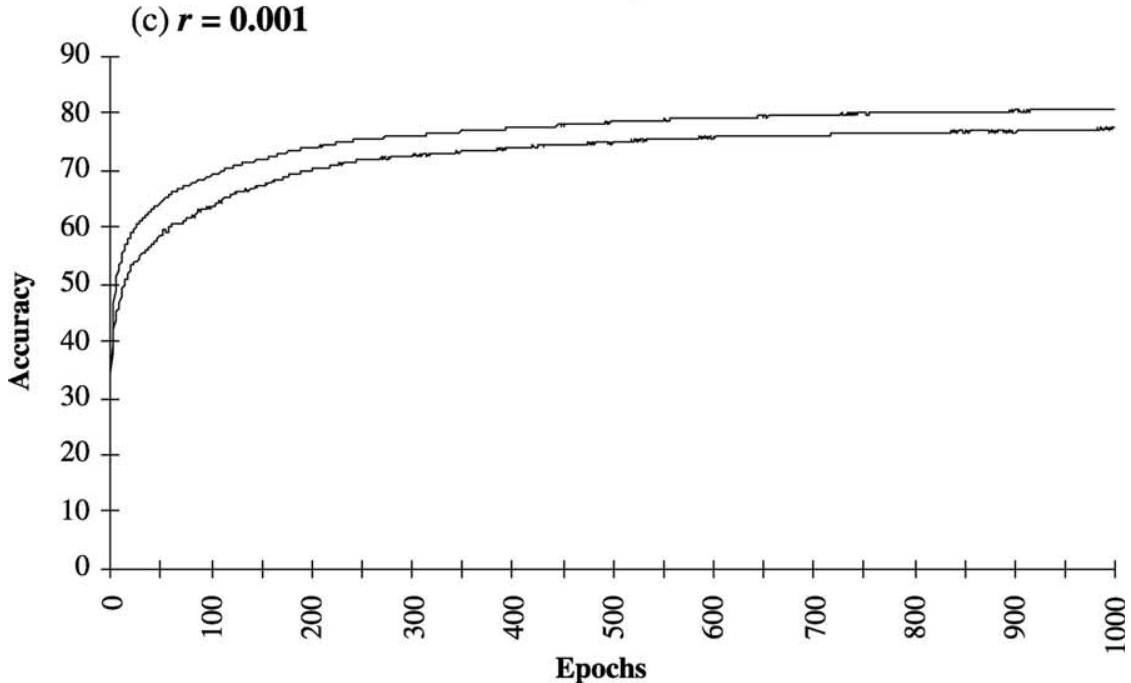

Fig. 6. Average generalization accuracy of on-line and batch training on 26 MLDB classification tasks, using learning rates of (a) 0.1 , (b) 0.01 , (c) 0.001 , and (d) 0.0001 . 
(d) $r=0.0001$

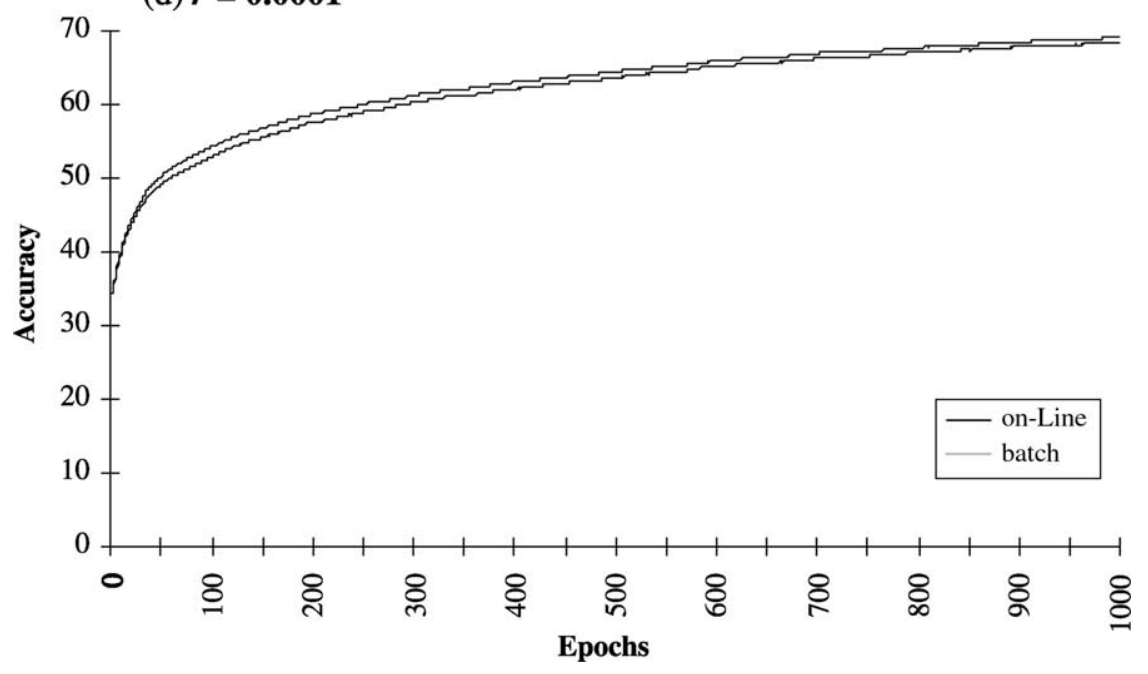

Fig. 6 (Continued)

the training set. For that task, a learning rate of 0.0001 was still too large for batch training, though on-line training did fine with a learning rate of 0.001 .

Table 1 summarizes the experiments on all of the Machine Learning Databases. The first two columns after the task's name show how many output classes each database had and the number of instances in the training set. The tasks are sorted by the size of the training set.

The main question was how long it took to train each task using on-line vs. batch training. To determine this for each task, the following steps were taken. For each learning rate $r$ in $\{0.1,0.01,0.001,0.0001\}$ and method $m$ in $\{$ on-line, batch\}, the average generalization accuracy on the hold-out set for all 10 trials after each training epoch was recorded, as was done for the Mushroom example in Fig. 5. The maximum accuracy for each such run was recorded, along with the number of epochs required to achieve that accuracy and the additional test set accuracy at that epoch.

Space does not allow the presentation of all of these results, but they are available in the on-line appendix (see Appendix A of this paper). Instead, the highest accuracy achieved by each method for any learning rate is shown under the heading 'Max Accuracy' in Table 1. The difference between the maximum accuracy for the on-line and batch training methods is shown under the heading 'Diff.'

The 'best' learning rate was chosen to be the one that used the fewest number of training epochs to get within $0.5 \%$ of the maximum accuracy achieved on that task by the respective method. For example, in the Pima Indians Diabetes task, on-line training achieved an accuracy of $76.41 \%$ after 287 epochs with a learning rate of 0.01 , but required 7,348 epochs to reach a maximum of $76.51 \%$ with a learning rate of 0.0001 . The difference in accuracy is not significant, but the difference in training time is dramatic, so the idea is to choose whichever learning rate achieves a reasonably good solution most quickly rather than depending so heavily on minor accuracy fluctuations.

On the other hand, on many tasks the maximum accuracy reached with a large learning rate was very poor, so such learning rates could not be considered best even though they reached their (poor) maximum very quickly. For example, on the Mushroom task, batch training achieved a maximum accuracy of $58.9 \%$ in just 2 epochs with a learning rate of 0.1 , but was able to get $100 \%$ accuracy in 1852 epochs using a learning rate of 0.0001 . In this case we obviously prefer the learning rate which produces substantially higher accuracy, even if it is much slower.

The best learning rate for each task is shown under the column 'Best LR' in Table 1. The number of epochs needed to reach the maximum accuracy by the best learning rate for each method is shown under the heading 'Epochs Needed.'

The last column of Table 1 shows what the speedup of on-line training was over batch training, which is how many times faster on-line training was than batch training on that task. The Shuttle and Letter Recognition tasks both required more than 10,000 epochs for batch training to converge. In both cases, plotting the results up to 10,000 epochs on a logarithmic scale showed that the best learning rate for batch training was progressing approximately 100 times slower than on-line training. It was not practical to run these tasks for the estimated 500,000-10,000,000 epochs they would need to reach their maximum.

On this set of 26 applications, on-line training was an average of over 20 times as fast as batch training. On applications with over 1000 training instances, on-line training was over 70 times as fast as batch training on average. On-line training was faster on all but one of the tasks in this set of tasks. In that one case, the Heart task, on-line training had a generalization accuracy that was $1.3 \%$ higher than batch, but required 825 epochs to achieve this, indicating that a learning rate of 0.1 is too 
Table 1

Summary of MLDB experiments. The maximum average generalization accuracy for on-line and batch training for each task are shown, along with the 'best' learning rate and the number of epochs required for convergence with the learning rate shown. The ratio of required epochs for batch to on-line training is shown as the 'speedup' of on-line training

\begin{tabular}{|c|c|c|c|c|c|c|c|c|c|c|}
\hline \multirow[t]{2}{*}{ Dataset } & \multirow[t]{2}{*}{ Output classes } & \multirow[t]{2}{*}{ Training set size } & \multicolumn{3}{|c|}{ Max accuracy } & \multicolumn{2}{|l|}{ Best LR } & \multicolumn{2}{|c|}{ Epochs needed } & \multirow[t]{2}{*}{ On-line's speedup } \\
\hline & & & On-line & Batch & Diff. & On-line & Batch & On-line & Batch & \\
\hline Bridges & 7 & 42 & 82.14 & 82.86 & -0.72 & 0.1 & 0.1 & 29 & 156 & 5.4 \\
\hline Hepatitis & 2 & 48 & 93.75 & 93.75 & 0.00 & 0.01 & 0.01 & 85 & 87 & 1.0 \\
\hline Zoo & 7 & 54 & 94.44 & 94.44 & 0.00 & 0.1 & 0.1 & 14 & 41 & 2.9 \\
\hline Iris & 3 & 90 & 100.00 & 100.00 & 0.00 & 0.01 & 0.01 & 596 & 602 & 1.0 \\
\hline Flag & 8 & 116 & 55.13 & 56.67 & -1.54 & 0.1 & 0.01 & 9 & 115 & 12.8 \\
\hline Sonar & 2 & 125 & 81.47 & 81.95 & -0.48 & 0.01 & 0.01 & 103 & 576 & 5.6 \\
\hline Glass & 7 & 128 & 72.32 & 73.02 & -0.70 & 0.1 & 0.01 & 921 & 6255 & 6.8 \\
\hline Voting & 2 & 139 & 95.74 & 95.74 & 0.00 & 0.1 & 0.01 & 6 & 49 & 8.2 \\
\hline Heart & 2 & 162 & 80.37 & 79.07 & 1.30 & 0.1 & 0.01 & 825 & 29 & $0.0^{\mathrm{a}}$ \\
\hline Heart (Cleaveland) & 5 & 178 & 85.33 & 84.33 & 1.00 & 0.01 & 0.01 & 80 & 500 & 6.3 \\
\hline Liver (Bupa) & 2 & 207 & 72.9 & 72.32 & 0.58 & 0.1 & 0.01 & 435 & 3344 & 7.7 \\
\hline Image segmentation & 7 & 252 & 97.86 & 97.5 & 0.36 & 0.1 & 0.01 & 305 & 2945 & 9.7 \\
\hline Vowel & 11 & 317 & 90.76 & 89.53 & 1.23 & 0.1 & 0.01 & 999 & 9434 & 9.4 \\
\hline CRX & 2 & 392 & 89.77 & 89.85 & -0.08 & 0.01 & 0.001 & 26 & 569 & 21.9 \\
\hline Breast cancer (WI) & 2 & 410 & 96.62 & 96.47 & 0.15 & 0.01 & 0.01 & 38 & 54 & 1.4 \\
\hline Australian & 2 & 414 & 88.41 & 88.41 & 0.00 & 0.001 & 0.0001 & 134 & 1257 & 9.4 \\
\hline Pima Indians diabetes & 2 & 461 & 76.41 & 76.73 & -0.32 & 0.1 & 0.01 & 58 & 645 & 11.1 \\
\hline Vehicle & 4 & 508 & 85.27 & 83.43 & 1.84 & 0.1 & 0.01 & 1182 & 9853 & 8.3 \\
\hline LED Creator & 10 & 600 & 74.35 & 74.05 & 0.30 & 0.1 & 0.01 & 29 & 179 & 6.2 \\
\hline Sat & 7 & 2661 & 92.02 & 91.65 & 0.37 & 0.01 & 0.001 & 3415 & 14049 & 4.1 \\
\hline Mushroom & 2 & 3386 & 100.00 & 100.00 & 0.00 & 0.01 & 0.0001 & 20 & 1820 & 91.0 \\
\hline Shuttle & 7 & 5552 & 99.69 & $97.44+$ & 2.25 & 0.1 & 0.0001 & 7033 & 9966 & $100.0^{\mathrm{b}}$ \\
\hline LED creator +17 & 10 & 6000 & 73.86 & 73.79 & 0.07 & 0.01 & 0.001 & 6 & 474 & 79 \\
\hline Letter recognition & 26 & 12000 & 83.53 & $73.25+$ & 10.28 & 0.001 & 0.0001 & 4968 & 9915 & $100.0^{\mathrm{b}}$ \\
\hline Average & 6 & 1329 & 86.76 & 86.15 & 0.62 & 0.061 & 0.014 & 842 & 2867 & 20.03 \\
\hline Median & 4 & 232 & 89.09 & 88.97 & 0.04 & 0.1 & 0.01 & 94 & 624 & 7.95 \\
\hline
\end{tabular}

a On the Heart task, on-line achieved $78.52 \%$ accuracy in 26 epochs with a learning rate of 0.01 .

b Batch training had not finished after 10,000 epochs for the Shuttle and Letter-Recognition tasks, but appeared to be progressing about 100 times slower than on-line.

large. Using a smaller learning rate of 0.01 , however, online training had an accuracy of $78.52 \%$ in 26 epochs, compared to $79.07 \%$ in 29 epochs for batch. Thus, even on this task, on-line was able to reach almost the same accuracy in slightly less time.

The speedup was dependent somewhat on the size of the training set, with the speedup being greater for larger training sets. Due to the variability between tasks, the relationship between training set size and speedup was not clear. From the above observations, it seems like it is typical to get an $\mathrm{O}(\sqrt{N})$ speedup when using on-line training, but further research is necessary to determine the relationship more precisely. Note that our experiments used learning rates that differed by factors of 10 in order to see overall trends in speedup. More precise relationships between training set size and speedup would require experiments using many learning rates along a more continuous scale. Regardless of the precise relationship, however, it is clear that on-line training is usually much faster than batch training on these tasks.

\subsection{Speech recognition task}

As mentioned above, in addition to on-line and batch training, it is possible to use mini-batch training with an update frequency $u, 1 \leq u \leq N$, where $u=1$ corresponds to on-line training (i.e. updating weights after each instance) and $u=N$ corresponds to batch training (i.e. accumulating over $u=N$ instances before updating weights). By using different values of $u$, it is possible to interpolate behavior between on-line and batch training to see what effect this has on training time and generalization accuracy at various learning rates. This in turn can yield insights into how the size of the training set affects batch training, since using an update frequency is similar to doing batch training on a 'batch size' of $u$.

A continuous digit speech recognition task was used to test the effect of the update frequency and learning rate on training speed and generalization accuracy. A multilayer perceptron with 130 inputs (plus one bias), 200 hidden nodes, and 178 outputs ( $=61,800$ weights) was trained on 
a training set of $N=20,000$ training instances. The output class of each instance corresponded to one of 178 contextdependent phoneme categories from a digit vocabulary. For each instance, one of the 178 outputs had a target of 1 , and all other outputs had a target of 0 . The targets of each instance were derived from hand-labeled phoneme representations of a set of training utterances.

To measure the accuracy of the neural network, a holdout set of 135 multi-digit speech utterances was run through a speech recognition system, for a total of 542 digit 'words.' The system extracted 130 input features per $10 \mathrm{~ms}$ frame from the speech signal and fed them into the trained neural network which in turn output a probability estimate for each of the 178 context-dependent phoneme categories on each of the frames. A dynamic programming decoder then searched the sequence of output vectors to find the most probable word sequence allowed by the vocabulary and grammar.

The word (digit) sequence generated by the recognizer was compared with the true (target) sequence of digits, and a dynamic programming algorithm was used to determine the number of correct matches, insertions, deletions, and substitutions in the utterance. The generalization word accuracy was computed as the percentage of correctly recognized words minus the percentage of additional inserted words, as is typical in speech recognition experiments (Rabiner \& Juang, 1993).

Mini-batch training was done with update frequencies of $u=1$ (i.e. on-line training), 10, 100, 1000, and 20,000 (i.e. batch training). Learning rates of $r=0.1,0.01,0.001$, and 0.0001 were used with appropriate values of $u$ in order to see how small the learning rate needed to be to allow each size of mini-batch training to learn properly.

For each training run, the generalization word accuracy was measured after each training epoch so that the progress of the network could be plotted over time. Each training run used the same set of random initial weights (i.e. using the same random number seed), and the same random presentation orders of the training instances for each corresponding epoch.

Training on 20,000 instances with 61,800 weights took much longer per epoch than any of the Machine Learning Database tasks, so it was not practical to run 10 trials of each series (i.e. each learning rate and batch size). Therefore, only one trial was run for each series. Furthermore, each series was run for up to 5000 epochs, but was stopped early if it appeared that the maximum accuracy had already been reached.

Table 2 summarizes the maximum accuracy achieved by each combination of batch size $u$ and learning rate $r$, as well as the number of training epochs required to achieve this maximum.

Using a learning rate of $r=0.1$ was slightly too large for this task, even for on-line training (i.e. $u=1$ ). It required almost as many training epochs as using a learning rate that was 10 times smaller (i.e. $r=0.01$ ), probably because it
Table 2

Summary of maximum hold-out set generalization word accuracy and training epochs required for each learning rate and batch size on the Digit speech recognition task

\begin{tabular}{lrlc}
\hline Learning rate & Batch size & Max word accuracy $(\%)$ & Training epochs \\
\hline 0.1 & 1 & 96.49 & 21 \\
0.1 & 10 & 96.13 & 41 \\
0.1 & 100 & 95.39 & 43 \\
0.1 & 1000 & $84.13+$ & $4747+$ \\
0.01 & 1 & 96.49 & 27 \\
0.01 & 10 & 96.49 & 27 \\
0.01 & 100 & 95.76 & 46 \\
0.01 & 1000 & 95.20 & 1612 \\
0.01 & 20,000 & 23.25 & $4865+$ \\
0.001 & 1 & 96.49 & 402 \\
0.001 & 100 & 96.68 & 468 \\
0.001 & 1000 & 96.13 & 405 \\
0.001 & 20,000 & 90.77 & 1966 \\
0.0001 & 1 & 96.68 & 4589 \\
0.0001 & 100 & 96.49 & 5340 \\
0.0001 & 1000 & 96.49 & 5520 \\
0.0001 & 20,000 & 96.31 & 8343 \\
\hline
\end{tabular}

repeatedly overshot its target and had to do some amount of backtracking and/or oscillation before settling into a local minimum.

Using learning rates of 0.001 and 0.0001 (i.e. 10 and 100 times smaller than 0.01 , respectively) required roughly 10 and 100 times as many epochs of training to achieve the same accuracy as using 0.01 for on-line training. Also, using learning rates of $0.01,0.001$, and 0.0001 allowed the safe use of mini-batch sizes of 10, 100, and 1000 instances, respectively, with reasonable but slightly less accurate generalization achieved by batch sizes of 100, 1000, and 20,000, respectively.

Figs. 7-10 show the overall trend of the generalization accuracy of each of the above combinations of learning rate and batch size as training progressed. Each series is identified by its values for $u$ (update frequency or batch size) and $r$ (learning rate). For example, '1000-0.01" means $u=1000$ and $r=0.01$. The horizontal axis shows the number of training epochs and uses a log scale to make the data easier to visualize. The actual data was sampled in order to space the points equally on the logarithmic scale, i.e. each accuracy value in these figures represents the average of the accuracy over the range of epochs spanned by that portion of the graph. Therefore, these graphs do not always show specific accuracy peaks, but rather show overall trends in accuracy for each batch size and learning rate.

Fig. 7 shows the results when using a learning rate of 0.1 . In this case, $u=1$ and $u=10$ were roughly equivalent, but $u=100 \mathrm{did}$ poorly, and $u=1000 \mathrm{did}$ worse yet. The learning rate was slightly too large even for on-line training and did not quite reach the same level of accuracy as smaller learning rates, while requiring almost as many epochs. Fig. 7(b) shows the same results as in Fig. 7(a), but zooms in 

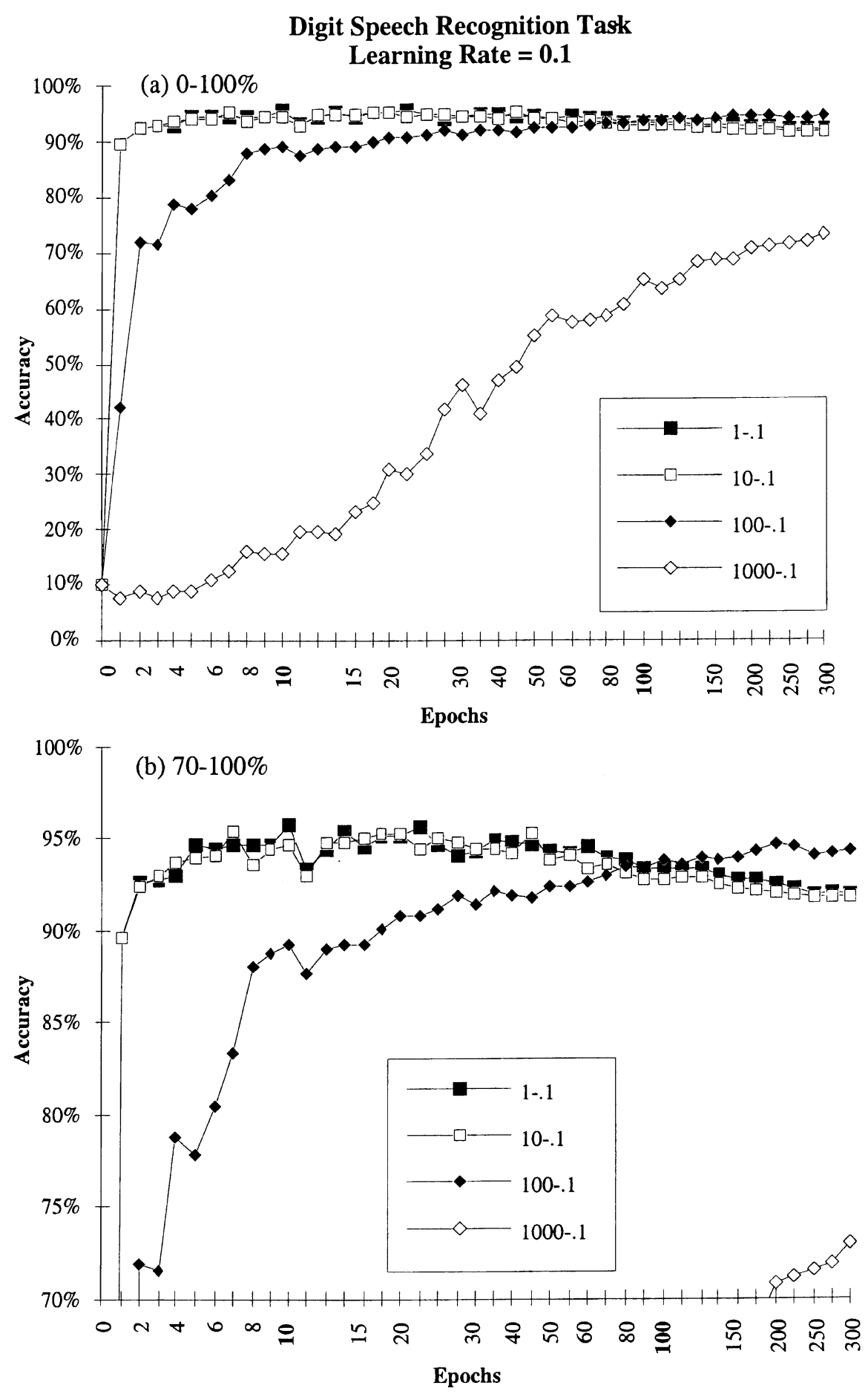

Fig. 7. Generalization accuracy on the Digit speech recognition task, using a learning rate of 0.1 . Each series represents a different mini-batch update frequency ( $1=$ on-line, '1000-0.1' accumulates changes over 1000 instances before updating weights).

on the higher accuracy range in order to show where there are subtle differences in accuracy between $u=1$ and $u=$ 10. Batch training was so slow for this learning rate that it was not plotted.

As shown in Fig. 8, when using the learning rate $r=$ $0.01, u=1$ and $u=10$ performed almost identically, achieving an accuracy of $96.49 \%$ after 27 epochs. Using $u=100$ was close but not quite as good $(95.76 \%$ after 46 epochs), and $u=1000$ did poorly (i.e. it took over 1500 epochs to achieve its maximum accuracy of $95.02 \%)$. Batch training performed no better than random for over 100 epochs and did not get above $17.2 \%$ accuracy after 5000 epochs.

Fig. 9 shows that for $r=0.001, u=1$ and $u=100$ performed almost identically, achieving close to the same 

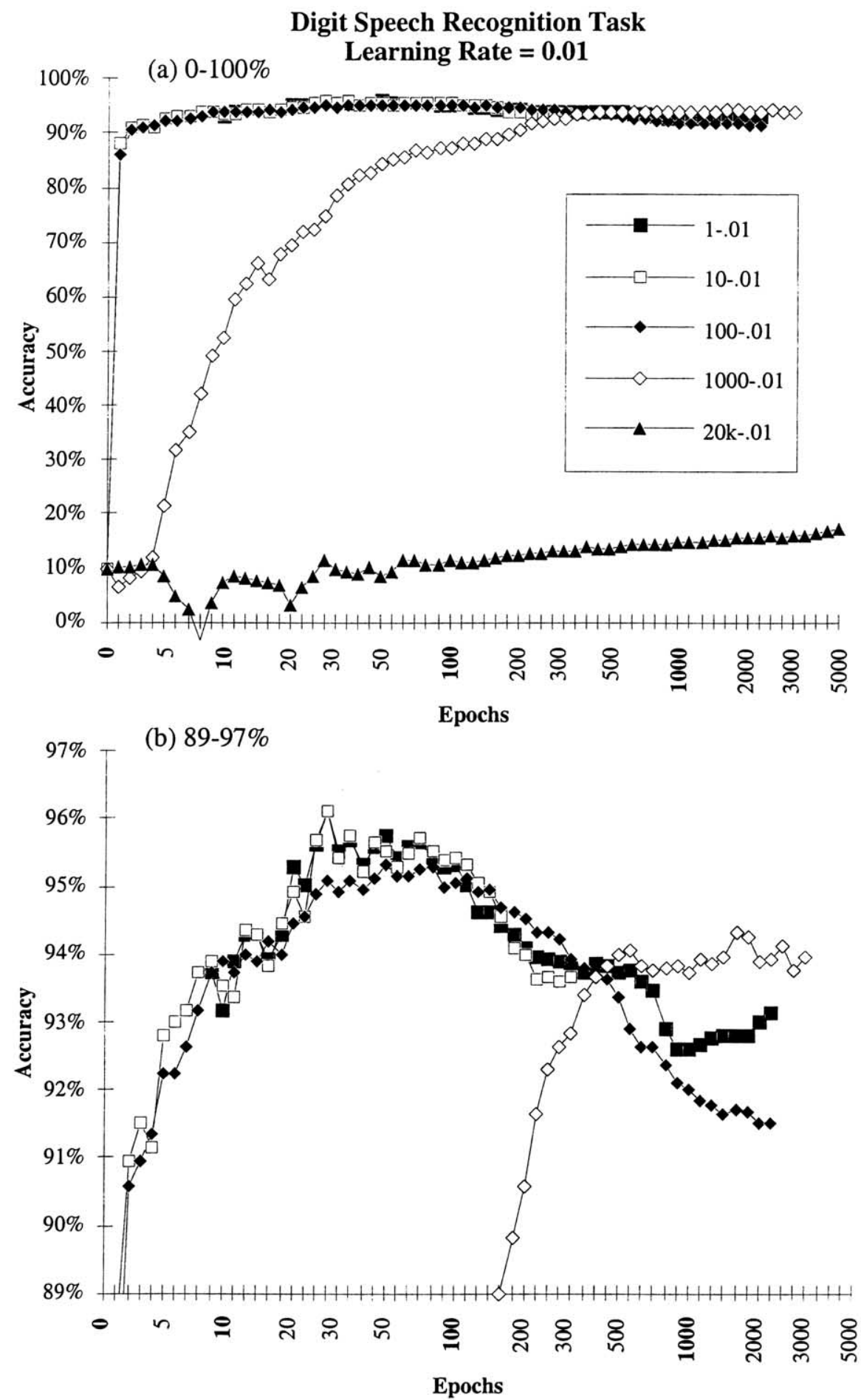

Fig. 8. Generalization accuracy on the Digit speech recognition task, using a learning rate of 0.01. Each series represents a different mini-batch update frequency $(1=$ on-line, $20 \mathrm{k}=$ batch $)$.

accuracy after about 400 epochs. (Since $u=10$ is between $u=1$ and $u=100$, which are almost identical for this learning rate, it is excluded for the sake of simplicity.) Using $u=1000$ caused a slight drop in accuracy, though it was close to the smaller values of $u$. As seen in Fig. 9(b), $u=1000$ followed a different path than the other two. Full batch training (i.e. $u=20,000)$ training did poorly, taking five times as long (1966 epochs) to reach a maximum accuracy of only $90.77 \%$.

Finally, using $r=0.0001, u=1, u=100$, and $\times$ $u=1000$ all performed almost identically, as shown in Fig. 10, achieving an accuracy of $96.31 \%$ after about 5000 

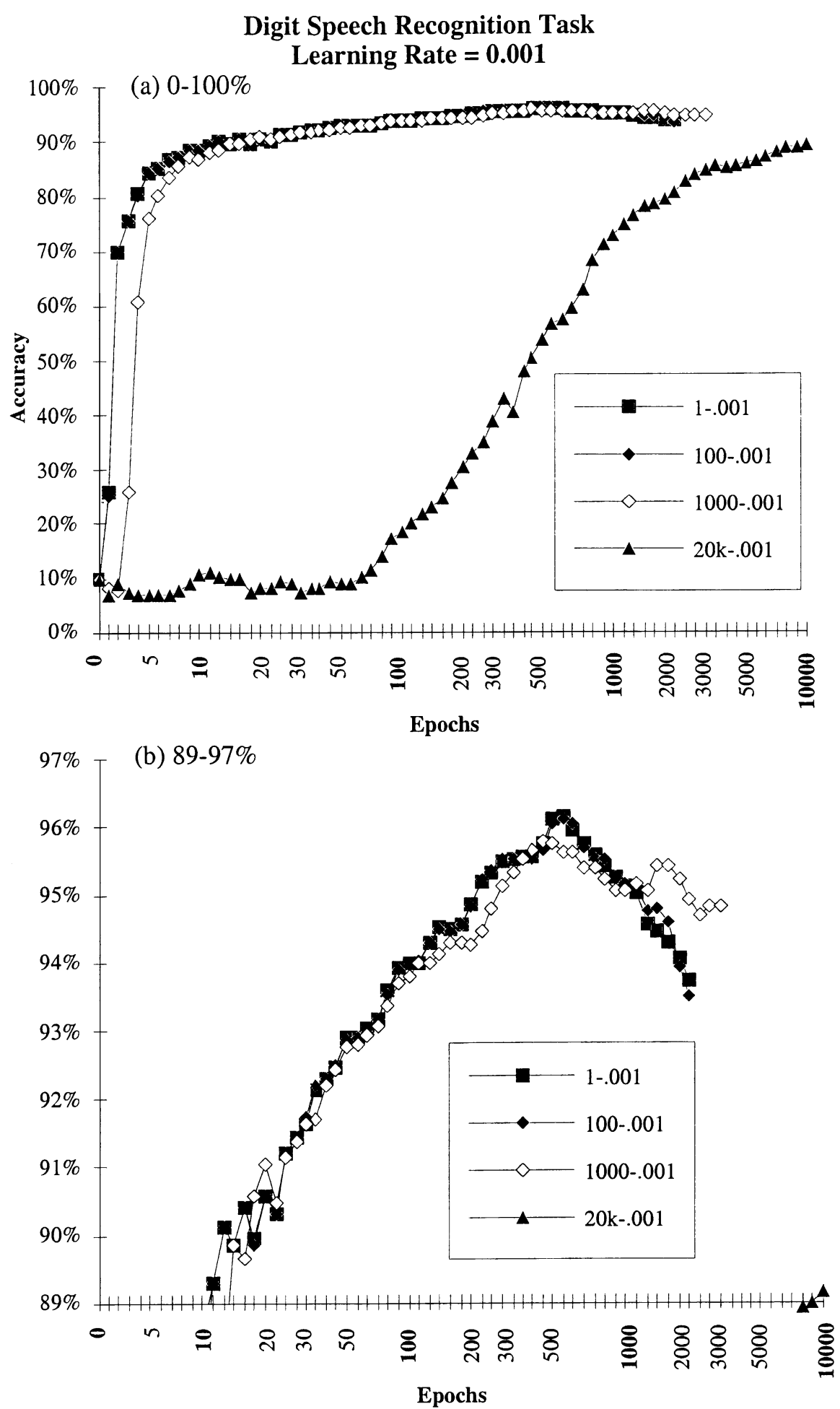

Fig. 9. Generalization accuracy on the Digit speech recognition task, using a learning rate of 0.001 .

epochs ( \pm 500 epochs). Batch training remained at random levels of accuracy for 100 epochs before finally training up to an accuracy that was just slightly below the others in 8343 epochs. Its accuracy lagged behind the others similar to how $u=1000$ did with $r=0.01$, indicating that a reduction of one or two more orders of magnitude on the learning rate might allow batch training to match on-line training more closely. Unfortunately, realistic constraints on floatingpoint precision and CPU training time did not allow the use of smaller learning rates. However, such results would 

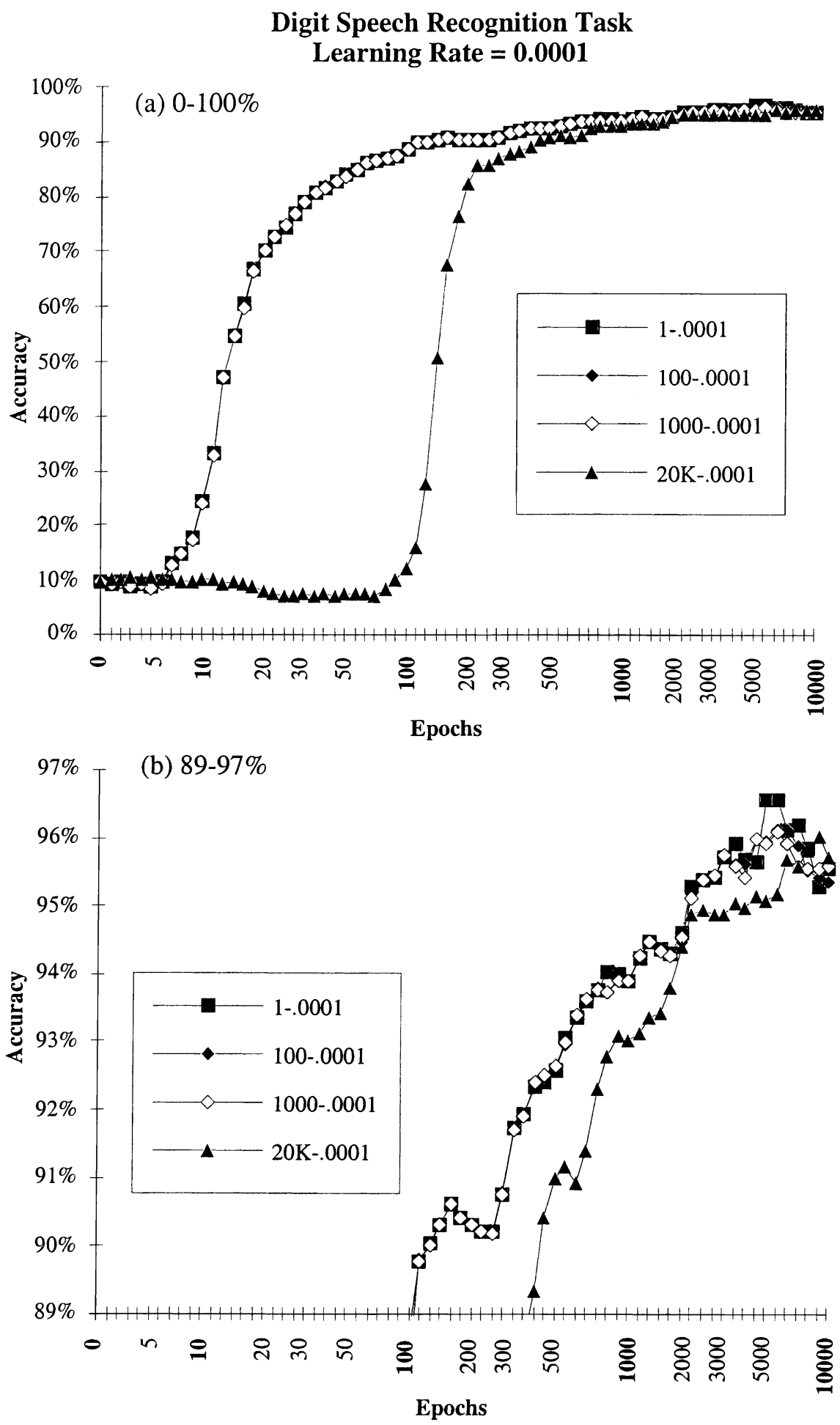

Fig. 10. Generalization accuracy on the Digit speech recognition task, using a learning rate of 0.0001 .

contribute little to the overall conclusions of the impracticality of batch training on large training sets.

Table 3 gives an overall summary showing how many training epochs were required to train the neural network using the best learning rate for each batch size $u$. In this table, the accuracy reported is that achieved on the test set, which was separate from the hold-out set used in Figs. 7-10 and Table 2. Table 3 shows that eventually, on-line, mini-batch and batch were able to achieve the same generalization accuracy, but that batch training required over 300 times as many epochs to do so.

These results illustrate that as soon as the learning rate is small enough to avoid drastic overshooting of curves and local minima, there is a linear relationship between 
Table 3

Summary of the best learning rate, test set generalization accuracy and training epochs required for each batch size on the Digit speech recognition task

\begin{tabular}{lllr}
\hline $\begin{array}{l}\text { Batch } \\
\text { size }\end{array}$ & $\begin{array}{l}\text { Best learning } \\
\text { rate }\end{array}$ & $\begin{array}{l}\text { Test set word } \\
\text { accuracy }(\%)\end{array}$ & $\begin{array}{c}\text { Training } \\
\text { epochs }\end{array}$ \\
\hline 1 & 0.01 & 96.13 & 27 \\
10 & 0.01 & 96.13 & 27 \\
100 & 0.001 & 96.12 & 468 \\
1000 & 0.0001 & 96.10 & 5520 \\
20,000 & 0.0001 & 96.04 & 8343 \\
\hline
\end{tabular}

the learning rate and the number of epochs required for learning. More importantly, they support the hypothesis that the more weight changes accumulated before being applied, the smaller the learning rate needs to be, and thus the longer training takes. Furthermore, they show no advantage in terms of either speed or accuracy for mini-batch or batch training.

\subsection{Problems with parallelism}

This research began as an experiment to determine how much faster a single neural network (i.e. multilayer perceptron trained via backpropagation) could be trained using a cluster of computers each operating on a separate partition of the training set. The final result was that largegrained parallelism (which requires batch or mini-batch training) is slower than training on a single machine using on-line training.

Given $M$ machines training on a training set of $N$ instances, each machine trains on approximately $N / M$ instances during each epoch, and shares its accumulated weight changes at the end. This must be done using batch training (or mini-batch training, using $u / M$ instances for each machine and synchronizing weights after every $u$ weight updates) since the weight changes are accumulated separately. Sharing accumulated weight changes would require $X \cdot \log 2 M$ time per epoch using a parallel gather operation, where $X$ is how many local weight updates can be done in the same amount of time as communicating a set of weight changes from one machine to another.

In the Digit experiments, batch training required over 300 times as many epochs as on-line training. Thus, 300 machines working together doing batch training in parallel would still be slower than a single machine using on-line training. Furthermore, the broadcast and gather operations required for synchronizing weight changes would be costly in terms of time under most communication architectures, in addition to the expense of using so many machines in the first place.

The larger the training set is, the slower batch training becomes with respect to on-line training, and thus the more likely it becomes that large-grained parallel training will actually be slower than serial on-line training. This is unfortunate, because large problems are exactly where training speed becomes most crucial. However, it is important to realize that large-grained parallelism will not typically help in training using batch (or mini-batch) gradient descent algorithms, but will in fact make training even slower than it already is.

On a more positive note, fine-grained parallelism (i.e. vector processors or other means of speeding up the weight and activation multiplication and summing) can speed up neural network training regardless of whether batch or on-line training is used, and is thus equally useful for both.

Finally, while large-grained parallelism turns out to not be useful for gradient descent algorithms, it can still be useful for many other kinds of training algorithms, including alternate algorithms for training multilayer perceptron and other neural networks. For example, an algorithm which stochastically searches a weight space for a multilayer perceptron (rather than using gradient descent) could benefit from having more machines looking at the weight space at the same time, with the best set of weights being occasionally shared between clusters.

\subsection{Future research}

While this paper does show that on-line training is much more efficient than batch training, further research is needed to establish a more precise relationship between training set size and the amount of speedup to expect from on-line training. In addition, although preliminary results indicate that the same overall conclusions hold when using a momentum term (e.g. as in backpropagation), it is not yet clear what the precise effect of the momentum term is on on-line's speedup. These questions may be difficult to answer precisely, since speedup is quite application-dependent.

Some researchers have suggested that the learning rate should be reduced over time in order to guarantee convergence of on-line training (Bishop, 1997; Reed \& Marks, 1999). However, some also suggest that "an aggressive (but subcritical) learning rate may produce faster learning even though it allows the error to increase occasionally" (Reed \& Marks, 1999). Our experiments used a constant learning rate, so further research is needed to determine whether it is advantageous to reduce the learning rate over time in practice, as well as how quickly it should be reduced.

The experiments in this paper used error backpropagation to train multilayer perceptrons. While we believe that the conclusions apply to most gradient descent algorithms, further experiments on other gradient descent algorithms would help to verify this assumption. 


\section{Conclusions}

A variety of common learning algorithms use gradient descent learning, which can be trained via batch or on-line training. Some neural network practitioners have been operating under the false assumption that batch training is as fast or faster than on-line training, and/or that batch training is more correct. In this paper we have explained that on-line training learns faster than batch training because it takes many steps per epoch which can follow curves in the gradient. On-line training handles large training sets and redundancy in data well and avoids the need to store an accumulated weight change.

Batch training, on the other hand, can only take a single step per epoch, which must be in a straight line. It estimates the gradient only at the starting point in weight space, and thus cannot follow curves in the error surface. As the size of the training set gets larger, batch training must use a smaller learning rate in order for its learning to remain stable.

At sufficiently small learning rates, on-line and batch training are essentially equivalent, but on-line training can safely use a larger learning rate than batch training, which allows it to reach the same level of accuracy in less time. No evidence has been found in this research to suggest that batch training is any more accurate than on-line training. On the contrary, at any given learning rate, on-line typically achieves accuracy that is as good (at small learning rates) or significantly better (at higher learning rates) than batch training.

The claim that batch training is more theoretically correct is misleading. While it is true that batch training calculates the true error gradient on the training set, the gradient is only accurate at the point of evaluation. When the learning rate is small enough for batch training to closely follow the gradient, on-line training will also follow the gradient as closely (or more so). When the learning rate is larger, both methods must use estimates of the gradient as they actually move through weight space. As each instance is presented, the expected value of the weight adjustment for on-line training is in the direction of the gradient at the current point, whereas for batch training it is in the direction of the gradient at the beginning of the epoch. Thus on-line training is usually a better approximation of a true gradient descent optimization.

In experiments on 26 classification tasks, on-line training was an average of over 20 times faster than batch training, and an average of over 70 times faster on tasks with over 1000 instances in the training set. On a large speech recognition task with 20,000 training instances, batch training took over 300 times as long as on-line training, and the required training time increased as the batch size grew larger in mini-batch training.

Current research and theory is demonstrating that for complex problems, larger training sets are crucial to avoid overfitting and to attain higher generalization accuracy. However, the problems with batch training are especially pronounced on large training sets. There appears to be no real advantage to batch training and a huge disadvantage in terms of efficiency. Therefore, we conclude that batch training is not a practical approach for gradient descent training on complex problems.

\section{Appendix A. An on-line appendix is available at the following location:}

\section{http://axon.cs.byu.edu/ randy/pubs/nn/batch}

This site contains complete experimental results, including the hold-out and test set accuracy for all trials on the Machine Learning Database tasks, as well as the hold-out and test set accuracy for all of the Digit experiments.

\section{References}

Atiya, A. F., \& Parlos, A. G. (2000). New results on recurrent network training: unifying the algorithms and accelerating convergence. IEEE Transactions on Neural Networks, 11-3, 697-709.

Barto, A. G. (1992). Reinforcement learning and adaptive critic methods. In D. A. White, \& D. A. Sofge (Eds.), Handbook of intelligent control. New York, NY: Van Nostrand Reinhold.

Becker, S., \& LeCun, Y. (1989). Improving the convergence of backpropagation learning with second order methods. In D. Touretzky, G. Hinton, \& T. Sejnowski (Eds.), (pp. 29-37). Proceedings of the 1988 Connectionist Models Summer School, Los Altos, CA: Morgan Kaufmann.

Bengio, Y (1991). Artificial neural networks and their application to sequence recognition, $\mathrm{PhD}$ Thesis, McGill University, Montreal, Quebec, Canada

Bengio, Y. (1996). Neural networks for speech and sequence recognition. New York: International Thomson Computer Press.

Bishop, C. M. (1997). Neural networks for pattern recognition. New York, NY: Oxford University Press.

Blake, C.L., Merz, C.J (1998). UCI Repository of Machine Learning Databases (www.ics.uci.edu/ mlearn/MLRepository.html). Irvine, CA: University of California, Department of Information and Computer Science.

Bouttou, L., Fogelman-Soulie, F., Blanchet, P., \& Lienard, J. S. (1990). Speaker independent isolated digit recognition: Multi-layer perceptrons vs. dynamic time-warping. Neural Networks, 3, 453-465.

Demuth, H., \& Beale, M. (1994). Neural network toolbox user's guide. Natick, MA: MathWorks, Inc.

Fausett, L. (1994). Fundamentals of neural networks. Englewood Cliffs, NJ: Prentice Hall.

Glasserman, P. (1991). Gradient estimation via perturbation analysis.

Hassoun, M. (1995). Fundamentals of artificial neural networks. Cambridge, MA: MIT Press.

Haykin, S. (1999). Neural Networks: a comprehensive foundation. Englewood Cliffs, NJ: Prentice Hall.

Kiefer, J., \& Wolfowitz, J. (1952). Stochastic estimation of the maximum of a regression function. Annals of Mathematical Statistics, 23, $462-466$.

Kohonen, T. (1982). Self-organized formation of topologically correct feature maps. Biological Cybernetics, 43, 59-69. 
Møller, M. F. (1993). A scaled conjugate gradient algorithm for fast supervised learning. Neural Networks, 6, 525-533.

Principe, J. C., Euliano, N. R., \& Lefebvre, W. C. (2000). Neural and adaptive systems. New York: Wiley.

Rabiner, L., \& Juang, B. H. (1993). Fundamentals of speech recognition. Englewood Cliffs, NJ: Prentice Hall.

Reed, R. D., \& Marks, R. J. (1999). Neural Smithing: supervised learning in feedforward artificial neural networks. Cambridge, MA: MIT Press.

Robbins, H., \& Monro, S. (1951). A stochastic approximation method. Annals of Mathematical Statistics, 22, 400-407.

Rosenblatt, F. (1962). Principles of neurodynamics: perceptrons and the theory of brain mechanisms. Washington: Spartan Press.

Rumelhart, D. E., \& McClelland, J. L. (1986). Parallel distributed processing. Cambridge, MA: MIT Press.
Sarle, Warren, S (2002) (current editor). comp.ai.neural-nets FAQ, part 2, available on-line at: ftp://ftp.sas.com/pub/neural/FAQ2.html

Spall, J. C. (1999). Stochastic optimization: stochastic approximation and simulated annealing. In J. G. Webster (Ed.), (Vol. 20) (pp. 529-542). Encyclopedia of electrical and electronics engineering. New York: Wiley.

Wasserman, P. D. (1993). Advanced methods in neural computing. New York, NY: Van Nostrand Reinhold.

Weiss, S., \& Kulikowski, C. (1990). Computer systems that learn. Los Altos, CA: Morgan Kaufmann.

Wilson, D. R., \& Martinez, T. R. (2001). The need for small learning rates on large problems. Proceedings of the 2001 International Joint Conference on Neural Networks (IJCNN'01), $115-119$. 